1 Long title: Host evolutionary history predicts virus prevalence across bumblebee

2 species

3 Short title: Evolutionary signals in bumblebee-virus interaction networks

4

5 David J. Pascall ${ }^{1, \# a^{*}}$, Matthew C. Tinsley ${ }^{2}$, Darren J. Obbard ${ }^{3,4}$ and Lena Wilfert ${ }^{1, \# b}$

6

$7{ }^{1}$ College of Life and Environmental Science, University of Exeter Cornwall Campus,

8 Treliever Road, Penryn, UK

$9 \quad{ }^{2}$ Biological and Environmental Sciences, Stirling University, Stirling, FK9 4LA, UK

$10{ }^{3}$ Institute of Evolutionary Biology, University of Edinburgh, Charlotte Auerbach

11 Road, Edinburgh, UK

$12{ }^{4}$ Centre for Infection, Evolution and Immunity, University of Edinburgh, Charlotte

13 Auerbach Road, Edinburgh, UK

$14{ }^{\# a}$ Institute of Biodiversity, Animal Health and Comparative Medicine, Graham Kerr

15 Building, University of Glasgow, Glasgow, UK

$16{ }^{\# b}$ Institute of Evolutionary Ecology and Conservation Genomics, University of Ulm,

17 Albert-Einstein-Allee 11, 89069 Ulm, Germany

18

$19{ }^{*}$ Corresponding author

20 Email: david.pascall@glasgow.ac.uk

21

Author Contributions 
DJP - Conceptualization, Data Curation, Formal Analysis, Investigation,

Draft Preparation, Writing - Review \& Editing

MCT - Investigation, Resources, Writing - Review \& Editing

DJO - Conceptualization, Data Curation, Formal Analysis, Funding Acquisition,

Methodology, Project Administration, Software, Visualisation, Writing - Review \&

Editing

31 LW - Conceptualization, Funding Acquisition, Investigation, Methodology, Project

Administration, Resources, Supervision, Writing - Review \& Editing

\section{Author's Summary}

Despite the importance of disease in the regulation of animal populations, our

understanding of the distribution of pathogen burden across wild communities

remains in its infancy. In this study, we investigated the distribution of viruses across natural populations of 13 different bumblebee species in Scotland. We first searched for viruses using a metatranscriptomic approach, finding at least 30 new viruses of bumblebees, and assayed a subset of them for their presence and absence in different host species. Then, in the first application of these methods to an animal-virus system, we used co-phylogenetic mixed models to investigate the factors that lead to species being having different prevalences for a subset of these viruses. While much of the variation in the prevalence of the viruses can be explained by the idiosyncrasies of individual bumblebee-virus pairings, there is a phylogenetic signal with related bumblebee species being infected at similar frequencies by the same sets of viruses. 

are more likely to exhibit similar patterns of infection.

\section{Abstract}

Why a pathogen associates with one host but not another is one of the most important questions in disease ecology. Here we use transcriptome sequencing of wild-caught bumblebees from 13 species to describe their natural viruses, and to quantify the impact of evolutionary history on the realised associations between viruses and their pollinator hosts. We present 37 novel virus sequences representing at least 30 different viruses associated with bumblebees. We verified 17 of them by PCR and estimate their prevalence across species in the wild. Through small RNA sequencing, we demonstrate that at least 10 of these viruses form active infections in wild individuals. Using a phylogenetic mixed model approach, we show that the evolutionary history of the host shapes the current distribution of virus/bumblebee associations. Specifically, we find that related hosts share viral assemblages, viruses differ in their prevalence averaged across hosts and the prevalence of infection in individual virus-host pairings depends on precise characteristics of that pairing.

\section{Introduction}

70 Pathogens that naturally infect more than one host species have a particularly high

71 risk of disease emergence [1]. One especially important group of pathogens are the

72 viruses, whose ubiquity leads them to have a disproportionate role in the regulation of 73 natural populations [2]. Viruses are relevant in populations that humans manage for 
economic and conservation reasons, such as bumblebees, which are both in decline [3] and important providers of ecosystem services [4].

Bumblebees, genus Bombus, are a primitively eusocial group of important wild pollinators. Many bumblebee species have experienced population declines, linked to biotic and abiotic stressors such as habitat degradation, pesticide use and shared infectious diseases for example caused by viral pathogens [5]. In contrast to honeybee viruses, which have been intensively studied, and have in many cases been found to represent multihost pathogens (see Manley et al (2015) [6] and the references within), bumblebee-specific viruses are comparatively poorly studied, and it is unknown how widely they are shared between host species.

For a species to be a multihost pathogen, some degree of opportunity for cross-species transmission must exist. Our definition of multihost pathogens follows that of Fenton et al. [7]. As such, multihost pathogens are defined to include two conceptually distinct groups: 'facultative multihost pathogens' that are able to maintain transmission chains in multiple host species (i.e. $R_{0}>=1$ in multiple host species) and 'obligate multihost pathogens', which rely on sufficiently high rates of cross-species transmission to offset unsustainable transmission within individual host species (i.e. $0<R_{0}<1$ within host species, $R_{0}>=1$ overall). In addition, pathogens that maintain transmission in a single host $\left(R_{0}>=1\right)$ but experience regular spillover (with or without the expectation of onward transmission: $0<=R_{0}<1$ ) are included as being effectively multihost pathogens within our definition. $R_{0}$ is defined as the expected number of secondary infections caused by a single typical infected individual in an entirely naïve host population [8]. We define cross-species transmission as the movement of a multihost pathogen between host species within its host range. This contrasts with 
host shifting, which we define as a transmission event to a new host species, leading

101 between cross-species transmission and host shifting in the case of pathogens that

102 exhibit rare spillover events.

104 The opportunity for cross-species transmission, which explains the large number of

105 viruses originally detected in honeybees that are present in bumblebees, may be

106 created by niche overlap in foraging [9]. Bumblebee nests are provisioned by foraging

107 workers who gather pollen and nectar from flowers in the surrounding area.

108 Considerable interspecific differences in plant species utilization by foragers of

109 different species are commonly observed [10-13], but this is not a universal

110 phenomenon [14], and the degree of overlap may depend on the diversity of flowers

111 currently in bloom. In bumblebees, flower choice of foragers is correlated with

112 species tongue length $[12,13]$, which implicitly incorporates shared behavioural

113 characteristics between closely related bumblebee species as there is phylogenetic

114 correlation between tongue length and relatedness [15]. Different species of

115 bumblebee also exhibit incomplete temporal separation throughout the year, causing

116 some degree of partitioning in niche space even when they are spatially sympatric

117 [16]. This ecology leads to a complex interaction network between bumblebee

118 species, as well as sympatric honeybees, which may structure cross-species

119 transmission.

121 The prevalence of pathogens, including viruses, across host species, such as

122 bumblebees, is structured on two levels. First, a virus may be present or entirely

123 absent in a potential host species. Second, other factors may then influence how 
124 prevalent a pathogen is within that species. At the presence/absence level, a complete

125 lack of infection in nature can occur in three ways: 1) a host and virus may exist in

126 allopatry or in completely non-interacting ecological niches, preventing transmission

127 irrespective of the host's susceptibility; 2) a physiological or molecular mismatch

128 (including immunity) between a host and virus can prevent infection; and 3)

129 environmental conditions may be such that transmission cannot occur between two

130 sympatric species. None of these mechanisms represent an immutable barrier, and all

131 represent ends of a continuum, where lesser forms simply reduce transmission.

132 Spatially or ecologically separated hosts and parasites may come into contact through 133 migrations or human facilitated invasions, allowing new associations to emerge. For

134 example, the arrival of Plasmodium relictum to the Hawaiian islands led to avian

135 population declines and contributed to extinctions in the naturally susceptible but

136 naïve populations [17]. Incompatibility can break down if evolution in the pathogen

137 or host removes the physiological or molecular barriers to infection, as shown when

138 Canine parvovirus type 2 emerged from Feline panleukopenia virus after gaining the

139 ability to bind to canine transferrin receptors [18].

140

141 For virus-host associations where infection can and does occur, quantitative

142 differences in infection risk between species can be driven by ecological variation in

143 transmission rates. These differences can be driven by, for example, the propensity for

144 group living [19], population densities [20], the biodiversity of the community [21]

145 and host avoidance behaviours [22]. Variation in infection risk among host species

146 can also be driven by physiological and molecular factors, with hosts having varying

147 suitability for the replication of a given parasite. In the extreme case, a host species

148 may exhibit condition-dependent susceptibility; where infection can only occur when 
149 the immune system is suppressed, either directly, through an immunosuppressant

150 disease or chemical agent, or indirectly, through trade-offs in resource allocation

151 brought about by malnutrition [23]. Both behavioural and ecological factors, leading

152 to differences in contact rate, and physiological factors, leading to differences in

153 infection probability on contact, may be phylogenetically correlated [15,24].

\section{Box 1 - Definition of Terms}

157 Co-phylogenetic generalized linear mixed models that incorporate phylogenetic

158 variance from multiple clades $[25,26]$ have been used relatively rarely, and a

159 biological interpretation of the model terms may not be immediately familiar. In the

160 host-parasite context, this approach can be used to model how the probability of

161 infection is predicted by both host and parasite species, allowing for covariance

162 induced by the relationships within each group, and the interactions between these

163 model terms. This can be considered either at the species-wide level (i.e. the

164 probability that infection will occur at all in a given host/parasite pairing), or at the

165 level of individuals within species (i.e. infection prevalence). Here we provide verbal

166 descriptions of how the terms can be interpreted, as well as references to a figure in

167 Hadfield et al. (2014) [25] where each of these effects is illustrated graphically:

169 Phylogenetic Effect: Variation in the mean value of a trait among species that is

170 explained by phylogenetic divergence. For example, more closely-related hosts might

171 be more similar in susceptibility to viral infection (display higher viral prevalence),

172 irrespective of virus species. Equivalently, more closely-related viruses might be

173 more similar in infectivity, irrespective of host species (Figs 1a and b in Hadfield et 
al. (2014) [25]).

175

176 Species Effect: Variation in the mean value of a trait among species that is

177 not explained by a Phylogenetic Effect. For example, much of the variation in

178 prevalence among viral species (irrespective of host), may not be explained by the

179 virus phylogeny but instead depend on lineage-specific viral traits (Figs $1 \mathrm{~g}$ and $\mathrm{f}$ in

180 Hadfield et al. (2014) [25]).

182 Non-phylogenetic Interaction: The interaction term between host and parasite Species

183 Effects, such that variation in the mean value of a trait within particular host/parasite

184 pairings depends the specifics of the host and parasite involved in a way not affected

185 by their evolutionary divergence. For example, variation in prevalence between

186 particular pairings that is caused by the interaction between lineage-specific host and

187 viral traits (Fig 1h in Hadfield et al. (2014) [25]).

188

189 Coevolutionary Interaction: The interaction term between host and parasite

190 Phylogenetic Effects, such that variation in the mean value of a trait within particular

191 host/parasite pairings depends on the evolutionary divergence among species in both

192 host and parasite clades. For example, if the prevalence of infection is more similar

193 among pairings of closely-related hosts and closely-related parasites than would be

194 expected from the host and parasite phylogenies and species-means alone. (Fig 1e in

195 Hadfield et al. (2014) [25]).

196

197 Evolutionary Interaction: The interaction term between the host (or parasite)

198 Phylogenetic Effect and the partners' Species Effect, such that the variation in the 
mean value of a trait within particular host/parasite pairings depends on the partner species, but is not predicted by the evolutionary divergence between partners'

202 species. For example, if the similarity in viral prevalence for one virus species is

203 strongly predicted by the evolutionary divergence among hosts, but a completely 204 different relationship (unrelated to the evolutionary divergence among viruses) is seen 205 for other virus species. (Fig 1c and d in Hadfield et al. (2014) [25]).

208 A new multihost virus can arise in two ways, either through a virus species gaining a new host [27] (Fig 1a), or through a speciation event in a multihost virus (i.e. giving

210 rise to two sibling multihost virus species; Fig 1b). When novel multihost viruses are 211 generated through host shifting (Fig 1a), a 'host evolutionary interaction' effect (see

212 Box 1) can result, as the consistent switching of viruses (V) to hosts (H) closely

213 related to their ancestral host will lead to related hosts having correlated viral

214 assemblages. When novel multihost viruses arise through speciation, i.e. if the ability 215 to infect multiple hosts is an ancestral trait (Fig 1b), a 'virus evolutionary interaction'

216 effect can result (see Box 1) through the inheritance of the ancestral host range,

217 leading to the daughter virus species having correlated host assemblages. These

218 effects can also be generated in ecological time through mechanisms that lead to 219 biased cross-species transmission. 
a

\section{Single host infection ancestral state Host phylogeny}

b

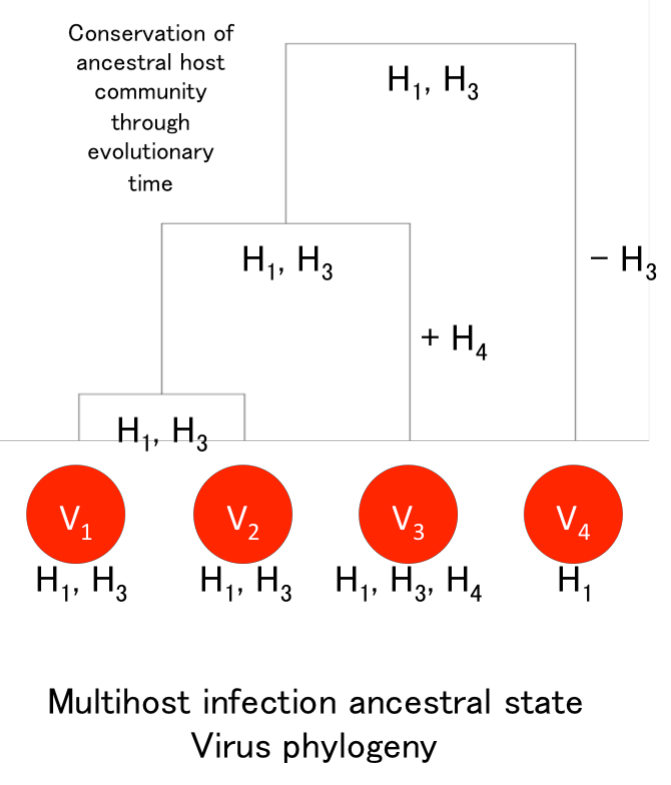

223 Fig 1 Mechanisms for the generation of novel multihost viruses. The generation of novel multihost viruses through host shifting (1a) leads to a 'host evolutionary interaction' effect (Box1), as the consistent switching of viruses $(\mathrm{V})$ to hosts $(\mathrm{H})$ closely related to their ancestral host will lead to related hosts having correlated viral assemblages. The generation of novel multihost viruses through speciation (1b) can lead to a 'virus evolutionary interaction' effect (Box 1) through the inheritance of the ancestral host range, leading to the daughter virus species having correlated host assemblages.

Here we test for a role of evolutionary history in shaping the current host/virus assemblage using species from an ecologically and economically important group, the bumblebees. We cataloged the virome of wild-caught bumblebees from across

233 Scotland by RNAseq, finding at least 30 new viruses. We then tested multiple

234 bumblebee species for a subset of these novel viruses and three previously reported

235 honeybee viruses: Slow bee paralysis virus [28], Acute bee paralysis virus [29] and

236 Hubei partiti-like virus 34 [30,31]. We analysed virus prevalence using co-

237 phylogenetic models to determine the presence or absence and relative strengths of 238 the phylogenetic signals that are expected to shape the host/virus assemblage in this system, and performed tests to attempt to determine the mechanisms driving this. 


\section{Methods}

\section{Sampling strategy}

243 A total of 926 individual bumblebees of 13 species were collected on the wing from

244 nine sites across Scotland in July and August of 2009 and 2011, and frozen in liquid 245 nitrogen or at $-80^{\circ} \mathrm{C}$. In 2009 , we sampled the Ochil Hills, Glenmore, Dalwhinnie, 246 Stirling, Iona, Staffa, and the Pentlands, and in 2011 we sampled Edinburgh and

247 Gorebridge (Supp Table 1). The cryptic species complex of Bombus terrestris,

248 Bombus lucorum, Bombus cryptarum and Bombus magnus was resolved using RFLP

249 analysis following Murray et al. (2008) [32]. All individuals were bisected

250 longitudinally prior to RNA extraction. One half of each bumblebee was used in

251 pooled RNA extractions of 2-11 individuals per species (median 10; Supp Table 2).

252 Two of these pools ('DIV' and 'P11') were included in the RNAseq, but excluded

253 from prevalence testing. The groups of bumblebees were ground in liquid nitrogen

254 and added to TRIzol reagent (Life Technologies) for RNA extractions, following the 255 manufacturer's standard protocol. The RNA concentrations in the pooled samples 256 were equalized to approximately $200 \mathrm{ng} / \mathrm{ul} /$ individual based on Nanodrop 257 measurements.

\section{RNA Sequencing and Bioinformatics}

260 The RNA was combined by species for B. terrestris (239 individuals), Bombus 261 pascuorum (212 individuals), B. lucorum (182 individuals) and other Bombus (293

262 individuals) into four large RNA pools. These large pools were sequenced using the 263 Illumina HiSeq platform with 100bp paired end reads (Beijing Genomics Institute) 264 after poly-A selection. This excludes ribosomal and bacterial RNA, and will enrich 
265 for mRNAs and those RNA viruses that have polyadenylated genomes or products.

266 The single-species bumblebee pools were subsequently re-sequenced following

267 duplex specific nuclease normalization, to reduce rRNA representation, and enrich for

268 rare transcripts while retaining non-polyadenylated viruses and products. The small

269 RNAs of the same RNA pools of B. terrestris, B. lucorum and B. pascuorum were

270 also sequenced to test for the replication of viruses identified via the transcriptome

271 sequencing.

273 For each pool, paired end RNAseq data were initially mapped to the published

274 Bombus terrestris and B. impatiens genomes using bowtie2 [33] to reduce the

275 representation of conserved bumblebee sequences. Read pairs that did not map

276 concordantly, including divergent bumblebee sequences and other associated

277 microbiota, were assembled de novo using Trinity 2.2.0 [34] as paired end libraries,

278 following automated trimming ('--trimmomatic') and digital read normalisation ('--

279 normalize_reads'). Where two RNAseq libraries (Poly-A and DSN) had been

280 sequenced, these were combined for assembly.

282 To identify putative viruses, all long open reading frames from each contig were

283 identified and concatenated to provide a 'bait' sequence for similarity searches using

284 Diamond [35] and BLASTp [36]. Contigs shorter than 500 base pairs were discarded.

285 These contig translations were used to search against a Diamond database comprising

286 all of the virus protein sequences available in NCBI database 'nr', and all of the

287 Dipteran, Hymenopteran, Nematode, Fungal, Protist, and prokaryotic proteins

288 available in NCBI database 'refseq protein' (mode 'blastp'; e-value 0.001; maximum

289 of one match). Matches to phage and short matches to large DNA viruses were 
excluded. Remaining contigs were manually curated to identify and annotate high-

291 confidence virus-like sequences. To quantify approximate fold-coverage, and to

292 assess viRNA properties, the raw RNAseq and trimmed small RNA reads were

293 mapped against the putative viral contigs using bowtie2's '--very-sensitive' setting

294 and retaining only the top map [33], from this we recorded the number of mapped

295 reads per kilobase of transcript per million mapped reads. We considered viruses to

296 show strong evidence of replication in the host if they had at least 50 mapping siRNA

297 reads with a size distribution sharply peaked at 22nt (viRNAs are generated from

298 replicating viruses by Dcr2). Following Fauquet and Stanley (2005) [37], we

299 designated contigs exhibiting less than $90 \%$ nucleotide identity as separate viruses

300 and those exhibiting greater than or equal to $90 \%$ identity as strains of known viruses.

\section{$302 \quad$ PCR Validation and Testing}

303 A subset of contigs were chosen for manual validation. All chosen contigs met both of

304 the following conditions: the presence of mapping reads in the bumblebee small

305 RNAs (for the B. terrestris, B. pascuorum and B. lucorum pools; not a condition for

306 the mixed Bombus pool) or the transcriptomic RNAs (for the other Bombus pool

307 where small RNAs were not generated), and the closest blast match being viral RNA-

308 dependent RNA-polymerase. Internal primers for these contigs were generated using

309 primer3 [38] and amplification of the target was verified via Sanger sequencing. See

310 Supp Table 3 for PCR conditions and primer sequences. Mayfield virus 1 and 2 were

311 Sanger sequence validated over the entirety of the contig. The Loch Morlich and

312 River Liunaeg virus sequences were generated by the connection of several disjoint

313 contigs by Sanger sequencing. Black Hill virus was excluded from further analysis as 
314 it was found that that the PCR reaction amplified a host sequence that could not be

315 visually differentiated from the virus product.

\section{Phylogenetic Inference}

318 Following Cameron et al. (2007) [39], we inferred the bumblebee phylogeny using

319 cytochrome oxidase I, elongation factor 1-alpha, opsin, phosphoenolpyruvate

320 carboxykinase, $16 \mathrm{~S}$ and arginine kinase genes. To break up long branches and allow

321 dating, additional species not sampled in the field were added from public databases

322 (see Supp Table 4 for genbank accession numbers and species included). The DNA

323 sequences were aligned with MAFFT using the L-INS-i setting [40,41]. The 6 gene

324 alignments were then used to generate the phylogeny in BEAST v2.4.5 [42], treating

325 each file as a separate partition, using bModelTest [43] with the

326 'transitionTransversion split' setting and a calibrated Yule tree prior [44]. An

327 uncorrelated lognormal relaxed clock was fitted to each partition, with exponential

$328(\lambda=1)$ priors placed over the mean rate and the default gamma $(\alpha=0.5396, \beta=0.3819)$

329 priors being placed over the standard deviation [45]. The bumblebees were

330 constrained to be monophyletic, with the honeybee, Apis mellifera, as an outgroup. A

331 gamma ( $\alpha=74.85889, \beta=0.4366812)$ distributed divergence time prior was placed

332 over the tMRCA of the Bombus clade, with parameters optimised to match the $2.5^{\text {th }}$

333 and $97.5^{\text {th }}$ percentiles of the posterior distribution of ages previously estimated by

334 Hines (2008) [46]. Four separate runs of the MCMC were performed for $100,000,000$

335 steps from random starting trees, with the first 50,000,000 steps being discarded as

336 burn in. Convergence of the posterior among runs was assessed in Tracer v1.7 [47].

337 The posterior sample was thinned to 1000 trees. 
339 For the virus phylogeny, amino acid sequences were inferred based on the translated

340 ORFs for regions predicted to contain RdRp motifs using the GenomeNet MOTIF

341 search function [48] against the Pfam database [49], with an expectation cut-off of

342 0.00001. If a virus had no annotated motifs, the canonical GDD RdRp amino acid

343 motif [50] was identified manually. Additional virus species (Supp Table 5) were

344 added to the phylogeny to anchor species with short generated contigs, and to break

345 up long branches. Given the long evolutionary distance between the viruses,

346 PROMALS3D [51] was used to align viral sequences. The alignments were trimmed

347 to the first conserved secondary structural element at both ends as predicted by

348 PROMALS3D with the 0.95 conservation metric. Two of the novel viruses (Agassiz

349 Rock virus and Cnoc Mor virus) were not included in this phylogeny because the

350 section of the RdRp gene required fell outside the available contig. Given that it is

351 unclear whether there was a universal common ancestor of all RNA viruses [52], we

352 aligned the sequences and generated the phylogeny twice, with and without the

353 negative sense RNA viruses (Supp Table 5). The trees serve purely to quantify

354 expected variance (under a Brownian motion model of evolution) between closely

355 related viruses. The deep splits in the phylogeny are poorly resolved with RdRp data

356 [53], due to the fast evolutionary rates of RNA viruses, the considerable time since

357 divergence and permutations in the RdRp sequence [54]. However, this should not

358 overly bias the conclusions as beyond a certain evolutionary distance, the viruses

359 would be expected to become essentially uncorrelated when averaged across the

360 posterior (Supp Table 6 for realised correlations).

362 Phylogenetic models used the BLOSUM62 rate matrix [55] with gamma distributed 363 rate variation using 4 gamma categories, an uncorrelated lognormal relaxed clock [45] 
364 and a Yule tree prior. A CTMC rate reference prior [56] was placed over the clock

365 mean and an exponential $(\lambda=1)$ prior was placed over the standard deviation. The

366 alpha parameter of the gamma distributed rate variation was given an exponential

$367(\lambda=1)$ prior. Absolute dating of viral trees is difficult due to the inconsistency in

368 estimated ages provided by estimated clock rates and known orthologous insertions

369 between sister host species [57], but is not essential for our analysis, which depends

370 only on relative branch lengths. Nevertheless, we chose to use orthologous insertions

371 (related historical viruses stably integrated into the genomes of species with better

372 known divergence dates) to provide approximate dates for our tree. To account for the

373 estimated ages of RNA viral families [58], we set a uniform lognormal prior with an

374 offset of 97 Mya, a mean of 500 Mya and a logged standard deviation of 0.5 on the

375 age of the root of the tree including the negative sense RNA viruses and a lognormal

376 prior with an offset of 76 Mya, a mean of 500 Mya and a logged standard deviation of

3770.5 on the age of the tree excluding them. Two partitiviruses (Rosellinia necatrix

378 partitivirus 2 and Raphanus sativus cryptic virus 1) known to have a common ancestor

379 older than 10 Mya [59] were included for dating purposes. We placed a diffuse

380 lognormal prior with an offset of 10 Mya, a mean of 30 Mya and a logged standard

381 deviation of 0.5 , on the age of the MRCA of these species. Both models were run over

38210 separate chains for 50,000,000 generations on a cluster in BEAST v1.8.4 [60],

383 with 25,000,000 generations being discarded as burn-in. Convergence of the posterior

384 was assessed in Tracer v1.7 [47]. The posterior distributions were combined and

385 thinned to 1000 trees. 
388 Maximum likelihood prevalence and 2-log-likelihood confidence intervals were

389 estimated for each host/virus combination with more than one pool using the code

390 from Webster et al. (2015) [61]. As the samples were small pooled groups of

391 individuals, such that a PCR 'positive' represents one or more infections, we

392 modelled the prevalence using a "pooled binomial" likelihood [62-64]. This approach

393 requires that the underlying prevalence of a virus is the same in all pools, which is

394 unlikely for bumblebees sampled from different locations. Estimates should therefore

395 be treated with caution.

\section{Co-phylogenetic Mixed Model Analysis}

398 To test for the evolutionary effects on association, the presence/absence data and the

399 phylogenetic trees were analysed using a co-phylogenetic mixed model [25]

400 implemented in Stan [65]. Our model is explicitly focused at the individual-level, and

401 the model's predictions represent the predicted probability of infection within an

402 individual of the species. This is in contrast with Hadfield et al.'s original

403 implementation where the focus was at the species- or population- level and the

404 model was estimating the probability that the parasite would be found in the species

405 or population at all. In all cases, the presented models showed no divergences,

406 acceptable Rhat and E-BFMI values and effective sample sizes of over 200.

407

408 We fitted host and virus phylogenetic effects, which measure the extent to which

409 variation in prevalence is clustered on the host and viral phylogenies respectively. We

410 also fitted host and viral evolutionary interaction effects, which measure the extent to

411 which related species have similar probabilities of infection in the sets of their

412 interaction partners. The final phylogenetic term fitted was a coevolutionary 
413 interaction, which measures the extent to which related hosts are infected to similar

414 degrees by related viruses (see Box 1).

416 In addition to the phylogenetic terms, non-phylogenetic host and virus terms, an

417 interaction between these terms and a pool ID term were fitted. The non-phylogenetic

418 host and virus terms measure variation that can be partitioned between host species

419 and virus species in average infection risk that is not consistent with trait evolution by

420 Brownian motion. The interaction term measures variation that can be partitioned

421 between pairwise interactions between individual hosts and viruses that is not

422 consistent with the linear sum of their individual means from the non-phylogenetic

423 host and virus terms. The pool ID effect measures variation between pools in

424 infection risk averaged over all the viruses tested. As the pools combined hosts by

425 species rather than by location, so that some had individuals from multiple locations,

426 we treated each location and each realised combination of locations as levels of a

427 random effect, terming this the "spatial composition effect". This describes the

428 variation in average infection level between realised combinations of locations

429 averaged across viruses. Model 1 included all the viruses, Model 2 excluded the

430 negative sense RNA viruses and Model 3 fitted a pseudo-taxonomic model. In Model

4313 , the relationship among the viruses was represented by a polytomic viral tree with

432 arbitrary branch lengths (with a root-to tip distance of 1 unit, and equal length

433 between each taxonomic level) with the viruses being split first by their genomic type

434 (+ve sense RNA, -ve sense RNA and dsRNA) implying a covariance of 0 between

435 genome structures, followed by splitting by the putative viral clades identified by Shi

436 et al. (2016) [31]. This was done to test for potential bias caused the by the possibility 
of systematic misidentification of the correct relationship between families in the estimated viral trees.

The form of the models is shown below, where $i$ indexes the data points, group $i$

441 represents the level of a categorical variable that the $i$ th pool belongs to, $y_{i}$ represents

442 the 1/0 indicator for the presence or absence of infection in the $i$ th pool, $k_{i}$ represents

443 the number of individuals in the $i$ th pool, $p_{i}$ is the unmeasured probability of infection

444 of a single individual in the $i$ th pool, $y_{i}^{\prime}$ is the estimated value of $\log _{e}\left(p_{i} /\left(1-p_{i}\right)\right), \mu$ is

445 the global mean of the latent variable, $\varepsilon$ is a normally distributed error term. All terms

446 were fitted as random effects (i.e. estimated by partial pooling). As above, a "pooled

447 binomial" likelihood was used [62-64].

$y_{i} \sim \operatorname{Bernoulli}\left(1-\left(1-p_{i}\right)^{k_{i}}\right)$

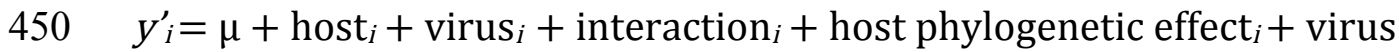

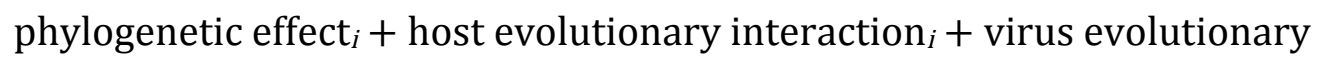

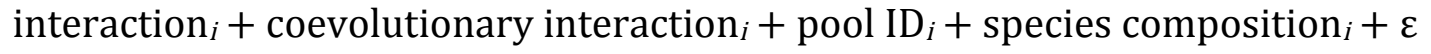

454 All variance-covariance matrices were generated as described in Hadfield et al.

455 (2014) [25], with the variance-covariance matrices scaled to correlation matrices. A

456 standard logistic prior was placed over the global intercept on the latent scale, $\mu$,

457 representing a flat prior on the probability scale. An exponential $(\lambda=1)$ prior was

458 placed on each variance term in the model. In the full model with all variances being

459 estimated, this is equivalent to a gamma $(\alpha=11, \beta=1)$ prior over the total variance,

460 which gives a prior mean variance of 11, and an appropriate prior on the standard 
461 deviation of a variable on the logit scale. Intraclass correlations, which represent the

462 proportion of the variance explained by each effect, were calculated on the link scale

463 (with an addition of $\pi^{2} / 3$ to the denominator to account for the variance of the logistic

464 distribution of the latent variable) from the model outputs and reported. Highest

465 posterior density intervals were calculated by the SPIn method [66] and $90 \%$ credible

466 intervals are reported as these are more robust to sampling in the tails of the posterior

467 distribution [67].

468

469 The total phylogenetic variance was calculated as:

470

$\left(\sigma^{2}\right.$ host phylogenetic $+\sigma^{2}$ host interaction $+\sigma^{2}$ virus phylogenetic $+\sigma^{2}$ virus interaction $+\sigma^{2}$ coevolutionary

interaction $) /\left(\sigma^{2}\right.$ total $\left.+\pi^{2} / 3\right)$

474 The total non-phylogenetic variance was calculated as:

$\left(\sigma_{\text {host }}^{2}+\sigma_{\text {virus }}^{2}+\sigma_{\text {interaction }}+\sigma^{2}\right.$ poolld $+\sigma^{2}$ spatial composition $) /\left(\sigma_{\text {total }}+\pi^{2} / 3\right)$

478 Uncertainty in the inferred phylogenies was accounted for by direct marginalisation.

479 This dramatically increased the runtime of the model, as, given the input of $H$ host 480 phylogenies and $V$ viral phylogenies from their posterior distributions, the likelihood 481 of each datapoint has to be calculated $H V$ times. As such, we included only 10 trees 482 from each posterior, as a trade-off between runtime and accounting for uncertainty in 483 the tree hypotheses. The marginalisation is shown below, with $\mathbf{y}$ being the total vector 484 of presences and absences, $H$ being the number of host phylogenies used, $V$ being the number viral phylogenies used, $\theta$ being all the non-variance-covariance parameters in 
the model, $\Omega_{j}$ being the set of variance-covariance matrices generated by the $j$ th combination of host and virus phylogenies, $\Omega_{H V}$ representing the set of all variancecovariance matrices being marginalised over and $\mathfrak{L}$ representing a likelihood.

$$
\mathfrak{L}\left(\boldsymbol{y} \mid \theta, \Omega_{H V}\right)=\sum_{j=1}^{H V} \frac{1}{H V} \mathfrak{L}\left(\boldsymbol{y} \mid \theta, \Omega_{j}\right)
$$

\section{Tongue Length Analysis}

After finding that the posterior for the host evolutionary interaction was well resolved from zero, we designed a post-hoc test to attempt to detect signal for one of the obvious mechanistic explanations for this; structured transmission networks driven by evolutionarily conserved anatomical factors. We tested for an association between the tongue length differences between bumblebee species and the differences in their viral community structures, as a proxy for signal of differential transmission at flowers driven by evolutionarily conserved flower choice. Average tongue lengths for each bumblebee species except Bombus bohemicus and Bombus cryptarum were taken from Goulson et al (2005) [68]. No published tongue length could be found for Bombus cryptarum, so we assumed that it was identical to that of Bombus lucorum, a species of which it is near indistinguishable in the field. Bombus bohemicus was excluded from this analysis, because it is an inquiline parasite, and therefore its ecology differs from the other species in such a way that tongue length would not be expected to be correlated with the viral community distance.

\section{In order to test for a correlation between tongue length and viral assemblage} similarity, estimates of the distance in viral communities between host species are required. These were generated as follows: For each host-virus combination, the 
511 package 'prevalence' was used to generate posterior draws of the underlying

512 prevalences under a Beta $(1,1)$ prior. Then 1000 draws per species were taken from

513 these sets of MCMC draws to generate 1000 matrices of host-virus prevalences

514 consistent with the raw data. For each of these matrices, the distance between each

515 species' viral community was calculated by taking the vector of estimated prevalences

516 for the 16 viruses of a given species as a coordinate in a 16-dimensional space then

517 calculating the Euclidean distance between these points. The rank correlation

518 (Kendall's $\tau$-b) between each pair of species' viral community distances and their

519 tongue length distances was then calculated, using the mantel function in the $\mathrm{R}$

520 package vegan. The point estimate presented is the median of the 1000 initial

521 correlations accounting for the uncertainty in the underlying prevalences. The $95 \%$

522 confidence interval is the $2.5^{\text {th }}$ and $97.5^{\text {th }}$ percentiles distribution of estimated

523 correlations.

525 Results

527 RNA was extracted from 13 species of bumblebee from nine sites, to identify new

528 viruses, assay their prevalence and their pattern of distribution across host species and

529 to test whether the evolutionary histories of the viruses and hosts have impacted the

530 current distribution.

532 Read and Assembly Statistics

533 A total of 134,026,056 sequencing read pairs were generated for Bombus lucorum,

534 135,590,922 for Bombus terrestris, 128,670,194 for Bombus pascuorum and

$53526,838,390$ for the other Bombus species with $0.37,0.38,3.36$ and 15.12 percent of 
reads mapping to the known viruses or the novel bee viruses found in the study. The poly-A and DSN normalized datasets were unexpectedly highly correlated, given their expected biases (1.000 for Bombus terrestris, 0.999 for Bombus pascuorum and 0.998 for Bombus lucorum) implying that the sequencing results were highly consistent irrespective of the selection method used.

\section{Previously Described Viruses Present in the Metagenomic Pools}

RNAseq reads mapped to three previously described bee viruses. The majority of these reads mapped either to the Acute bee paralysis virus/Kashmir bee virus complex (henceforth ABPV) [29] or to Slow bee paralysis virus (SBPV) [28]. Additionally, in the mixed Bombus pool, reads were found mapping to Hubei partiti-like virus 34 (HPLV34) a virus initially detected, though not named, in honeybees by Cornman et al (2012) [30], then subsequently also reported in a sample from Chinese landsnails by Shi et al (2016) [31].

No RNAseq reads were mapped to Deformed wing virus - type A [69], Chronic bee paralysis virus [29], Bee macula-like virus [70], Ganda bee virus [71], Scaldis River bee virus [71], Black queen cell virus [72], Apis rhabdovirus 1 [73], Apis rhabdovirus 2 [73], Apis bunyavirus 1 [73], Apis bunyavirus 2 [73], Apis flavivirus [73], Apis dicistrovirus [73], Apis Nora virus [73] and members of the Lake Sinai virus complex [74]. A small number of small RNA reads did map to these viruses, however, this likely represents cross-mapping, given the lower stringency of $22 \mathrm{nt}$ reads. Two of the viral contigs generated by the de novo assembly had high similarity to previously described plant viruses; both RNAs of White clover cryptic virus 2 [75] (96\% identity), both RNAs of strain of Arabis mosaic virus (MH614320/MH614321) [76] 
561 distant to previously sequenced strains (91\% identity) and a strain of Red Clover

562 nepovirus A (MH614312) [77] distant to previously sequenced strains (90\% identity).

563

\section{Putative Novel Viral-like Sequences}

565 We identified 37 putative novel viral contigs, four mapping to DNA viruses (4

566 densovirus-like contigs) and 33 to RNA viruses (4 Reo group contigs, 2 Toti-Chryso

567 group contigs, 4 Bunya-Arena group contigs, 1 Orthomyxoviridae-like contig, 8

568 Hepe-Virga group contigs, 12 Picorna-Calici group contigs and 2 Tombus-Noda

569 group contigs). Based on the supposition that a contig represents a separate virus if it

570 maps to a different viral grouping than the other contigs, or if it can be aligned to all

571 other contigs within its assigned viral grouping, this represents 30 new viruses with

572 seven remaining contigs that may represent other genomic regions of these 30 viruses

573 or separate viruses that cannot be confirmed as such. See Table 1 for information on

574 the viruses tested for prevalence using PCR and Supplementary Table 7 for detailed

575 information on all of the identified contigs. The numbers of reads mapping these

576 contigs were variable and are shown in Table 2.

578 Table 1 The names, genome structures and groupings (following Shi et al. (2016) [31]) of the newly

579 discovered viruses for which prevalence was assessed.

\begin{tabular}{llll}
\hline Putative viral contig & Abbreviations & Clade & Genome structure \\
\hline Agassiz Rock virus & ARV & Reo & dsRNA \\
Elf Loch virus & ELV & Reo & dsRNA \\
Dumyat virus & DV & Toti-Chryso & dsRNA \\
Sheriffmuir virus & SV & Toti-Chryso & dsRNA \\
Clamshell Cave virus & CCV & Bunya-Arena & - ssRNA \\
Allermuir Hill virus 1 & AHV1 & Hepe-Virga & +ssRNA \\
Allermuir Hill virus 2 & AHV2 & Hepe-Virga & +ssRNA \\
Allermuir Hill virus 3 & AHV3 & Hepe-Virga & +ssRNA \\
Mill Lade virus & MLV & Hepe-Virga & +ssRNA
\end{tabular}


bioRxiv preprint doi: https://doi.org/10.1101/498717; this version posted January 22, 2019. The copyright holder for this preprint (which was not certified by peer review) is the author/funder, who has granted bioRxiv a license to display the preprint in perpetuity. It is made available under aCC-BY 4.0 International license.

$\begin{array}{llll}\text { Boghill Burn virus } & \text { BBV } & \text { Picorna-Calici } & \text { +ssRNA } \\ \text { Gorebridge virus } & \text { GV } & \text { Picorna-Calici } & \text { +ssRNA } \\ \text { Loch Morlich virus } & \text { LMV } & \text { Picorna-Calici } & \text { +ssRNA } \\ \text { Mayfield virus 1 } & \text { MV1 } & \text { Picorna-Calici } & \text { +ssRNA } \\ \text { Mayfield virus 2 } & \text { MV2 } & \text { Picorna-Calici } & \text { +ssRNA } \\ \text { River Liunaeg virus } & \text { RLV } & \text { Picorna-Calici } & \text { +ssRNA } \\ \text { Castleton Burn virus } & \text { CBV } & \text { Tombus-Noda } & \text { +ssRNA }\end{array}$

581 Table 2 The RNAseq reads per kilobase per mapped million reads in the Bombus terrestris, Bombus

582 lucorum, Bombus pascuorum and mixed Bombus pools. Structural zeros are indicated by dashes, zeros

583 in the table indicate below 0.005. Contigs with names in bold meet the criterion of having at least 50

584 mapping small RNA reads with a sharp peak in the size distribution at 22nt in Bombus terrestris,

585 Bombus lucorum and Bombus pascuorum providing evidence of replication (see main text).

\begin{tabular}{|c|c|c|c|c|c|}
\hline Putative viral contig & $\begin{array}{c}\text { Accession } \\
\text { number }\end{array}$ & $\begin{array}{c}\text { Bombus } \\
\text { terrestris }\end{array}$ & $\begin{array}{l}\text { Bombus } \\
\text { lucorum }\end{array}$ & $\begin{array}{c}\text { Bombus } \\
\text { pascuorum }\end{array}$ & $\begin{array}{c}\text { mixed } \\
\text { Bombus }\end{array}$ \\
\hline Bombus-associated Densovirus-like Contig 1 & MH614322 & 0.09 & - & - & 12.34 \\
\hline Bombus-associated Densovirus-like Contig 2 & MH614323 & 0.39 & 2.66 & - & 14.23 \\
\hline Agassiz Rock virus & MH614287 & 3.77 & 0.49 & - & - \\
\hline Cnoc Mor virus & MH614297 & 0.97 & - & - & 20.38 \\
\hline Bombus-associated Reoviridae-like Contig 1 & MH614298 & 0.42 & 0.03 & - & 1.63 \\
\hline Elf Loch virus & MH614300 & - & 0.00 & 1.00 & 0.05 \\
\hline Dumyat virus & MH614299 & - & - & - & 10.30 \\
\hline Sheriffmuir virus & MH614317 & - & - & - & 2.55 \\
\hline Clamshell Cave virus & MH614294 & 0.07 & - & - & 2.10 \\
\hline Bombus-associated Bunyaviridae-like Contig 1 & MH614295 & 0.70 & - & - & 5.61 \\
\hline Bombus-associated Bunyaviridae-like Contig 2 & MH614296 & - & - & - & 12.13 \\
\hline Bombus-associated Phlebovirus-like Contig 1 & MH614315 & 3.07 & 2.77 & 0.95 & 1.63 \\
\hline Bombus-associated Orthomyxovirus-like Contig 1 & MH614314 & - & - & 0.44 & - \\
\hline Allermuir Hill virus 1 & MH614288 & 15.27 & 0.64 & 0.02 & 1.50 \\
\hline Allermuir Hill virus 2 & MH614289 & 0.01 & 0.03 & 12.71 & 0.13 \\
\hline Allermuir Hill virus 3 & MH614290 & 0.40 & 2.62 & 0.50 & 3.35 \\
\hline Mill Lade virus & MH614306 & 0.40 & 0.48 & 0.03 & 7.28 \\
\hline Bombus-associated Virga-like Contig 1 & MH614308 & - & 0.65 & - & - \\
\hline Bombus-associated Virga-like Contig 2 & MH614309 & - & 0.33 & - & - \\
\hline Bombus-associated Virga-like Contig 3 & MH614318 & 16.03 & 4.83 & 0.52 & 90.37 \\
\hline Bombus-associated Virga-like Contig 4 & MH614319 & 1.66 & 0.11 & - & 0.54 \\
\hline Black Hill virus & MH614291 & - & - & - & 2.96 \\
\hline Boghill Burn virus & MH614292 & 0.00 & 10.92 & 0.00 & 0.11 \\
\hline
\end{tabular}


Gorebridge virus

Bombus-associated Picornavirus-like Contig 1

Loch Morlich virus

Mayfield virus 1

Mayfield virus 2

Bombus-associated Nepovirus-like Contig 1

Bombus-associated Nepovirus-like Contig 2

Bombus-associated Picornavirus-like Contig 2

River Liunaeg virus

Castleton Burn virus

Bombus-associated Nodavirus-like Contig 1

\begin{tabular}{|c|cccc} 
MH614301 & 2.97 & 0.02 & - & 0.15 \\
MH614302 & 6.27 & 0.02 & - & 0.29 \\
MH614303 & 0.00 & - & - & 7.83 \\
MH614304 & 391.31 & 232.93 & 0.59 & 7.67 \\
MH614305 & 4.87 & 0.79 & 336.97 & 558.82 \\
MH614310 & 1.72 & 1.02 & 0.09 & 1.70 \\
MH614311 & 0.33 & 0.39 & 0.11 & 0.21 \\
MH614316 & 0.11 & 0.29 & 1.02 & 0.04 \\
MH614307 & 0.24 & 0.47 & 0.01 & 9.58 \\
MH614293 & 2.39 & 12.30 & 8.10 & 122.99 \\
MH614313 & - & - & - & 1.50
\end{tabular}

\section{siRNA-based Evidence for Infection} antisense mapping orientation being more prevalent. This 5' U-bias is consistent with

RNA interference is an important component of antiviral defence in arthropods [78].

As part of this defence mechanism, homologs of Drosophila Dicer-2 cleave dsRNA, usually in the form of replication intermediates, giving rise to a characteristically narrow and sharply peaked distribution of virus-derived small RNAs. Thus the presence of such small RNAs from both strands of an ssRNA virus provide compelling evidence that the virus was replicating. In bumblebees the characteristic Dicer-mediated viral siRNAs peak sharply at 22nt [73], and the viruses that displayed at least 50 characteristic viral siRNAs are marked in Table 2 . The distribution of the mapped small RNA reads is shown in Fig 3 for all viruses where the siRNAs are described in the main text, with full data in Supp Fig 1.

In the three bumblebee species with siRNA data, a sequence similar to a phlebovirus glycoprotein (AEL29653.1) displayed >50 siRNA reads. However, the size spectra of these reads is centered on $24 \mathrm{nt}$ with a strong bias for a 5' terminal uracil, with the insect piRNAs [79], and the predominant antisense orientation is consistent with the 
604 piRNA mapping pattern to endogenous viral elements (EVE) in mosquitoes [80].

605 However, the size of piRNAs in bumblebees is generally larger than this [81]. This

606 sequence is therefore potentially an EVE that has either been gained multiple times or

607 has been maintained in the bumblebee genome since at least the B. pascuorum- $B$.

608 terrestris/B. lucorum split.

609

610 It is notable that the size distribution of viral siRNAs is less sharply peaked in

611 Mayfield virus 1, Mayfield virus 2 and Slow bee paralysis virus (excepting Mayfield

612 virus 1 in B. lucorum, which is sharply peaked), with broad 'shoulders'. This is

613 reminiscent of the pattern seen for Drosophila C virus and Drosophila Nora virus in

614 wild-collected D. melanogaster [61], both of which contain a viral suppressor of

615 RNAi $[82,83]$.

616

617 B. pascuorum also had siRNA reads mapping to a sequence with $90 \%$ identity to Red

618 clover nepovirus A (MG253829.1) [77]. However, the read length spectra were

619 sharply peaked at $21 \mathrm{nt}$, rather than the $22 \mathrm{nt}$ of bumblebee viRNAs. This is consistent

620 with siRNA's produced from DLC4, the key antiviral dicer in Arabidopsis thaliana

621 [84] implying acquisition of the small RNAs through nectar or pollen contamination. 


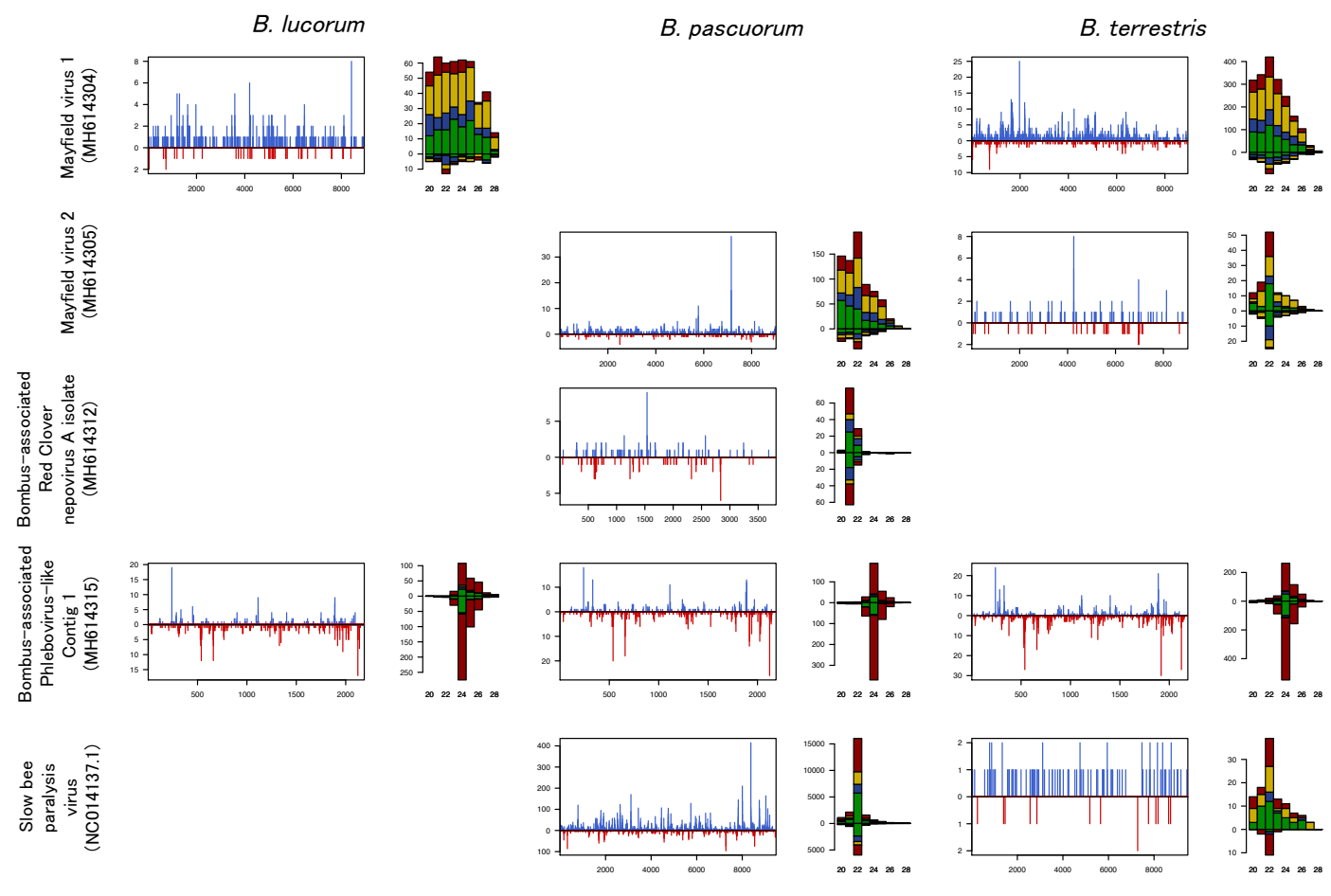

623

Fig 2 The mapping of small RNA reads to Mayfield virus 1, Mayfield virus 2, a contig similar to Tomato black ring virus, a contig similar to a phlebovirus glycoprotein and Slow bee paralysis virus. Blue lines represent reads mapping to the positive-sense strand at that genomic position, red lines represent reads mapping to the negative sense strand. The histogram of read size spectra shows the count of reads of each length mapping in the positive (above) and negative (below) directions. The colouring of each bar shows the counts of the reads beginning with each 5' base (red-U, blue-C, greenA, yellow-G).

\section{Prevalence}

Species level prevalences differed dramatically among the different viruses (Fig 3).

Prevalences were generally low to intermediate, with modal viral prevalences for most host-virus combinations being below $15 \%$. Slow bee paralysis virus was by far

636 the most common virus in the sample, with estimated prevalences of greater than $25 \%$

37 in multiple species. Our ability to estimate the prevalence of common viruses is

638 limited by the pooling, leading us to only be able to assign lower bounds to 
639 prevalences in these cases, but in 7 of 11 species, all pools were positive for SBPV.

640 Acute bee paralysis virus, Hubei partiti-like virus 34, Castleton Burn virus,

641 Gorebridge virus, Mayfield virus 1 and Mayfield virus 2 all reached 15-25\%

642 prevalences in multiple species. Several viruses showed strong signals of species

643 specificity, having very low to zero prevalences in multiple host species but high

644 prevalences in others. Examples of this pattern include Allermuir Hill virus 1 in $B$.

645 terrestris, Allermuir Hill virus 2 in B. pascuorum, Allermuir Hill virus 3 in B. magnus

646 and B. monticola, as well as Loch Morlich virus and River Luinaeg virus in $B$.

647 jonellus.

648

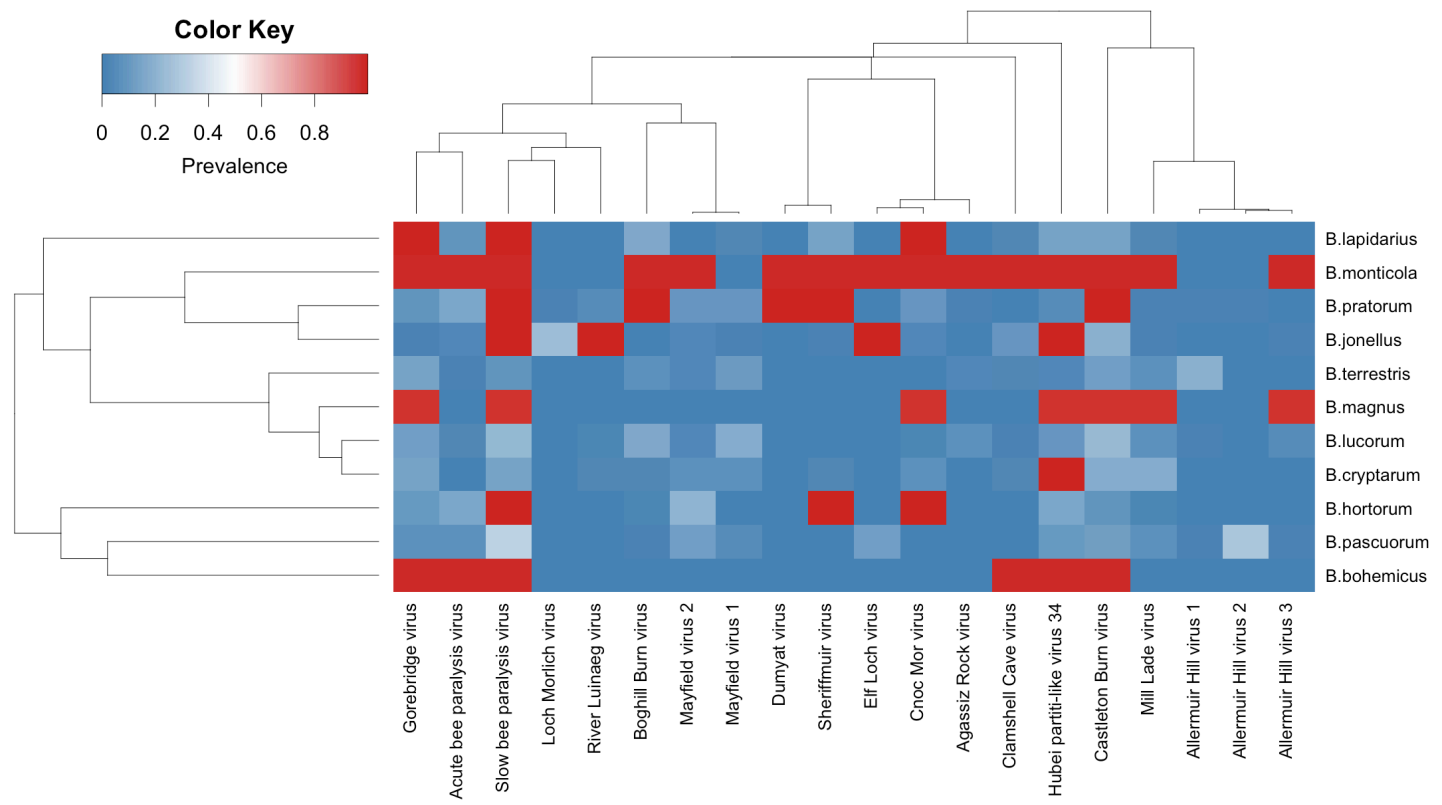

Fig 3 A heatmap of maximum likelihood estimates for prevalence. Hosts and viruses are ordered by

651 phylogenetic relatedness, the trees represent the maximum clade credibility topology. Squares in red

652 with maximum likelihood estimates of the prevalence of 1 correspond to cases where all pools were

653 positive. The maximum likelihood estimate is likely extremely upwardly biased in this case.

656 All models that included a virus phylogeny term gave qualitatively similar results (Fig 
657 4, Table 3). This suggests that the results are robust to both phylogenetic uncertainty

658 and the assumption of a common ancestor of all RNA viruses. For this reason, for the

659 rest of this section, estimates will be given from the model containing the estimated

660 phylogeny with all the RNA viruses included. All estimates represent the percentage

661 of the total variance in the model (the sum of all estimated variance components

662 adjusted for the variance of the link function by the addition of $\pi^{2} / 3$ ) explained by a

663 term. In all cases, the presented point estimate is the posterior mean, and $90 \%$ shortest

664 posterior density intervals [66] are presented following in square brackets. Shortest

665 posterior density intervals are a variant of highest posterior density intervals and

666 describe the shortest possible interval containing (in this case) $90 \%$ of the probability

667 density for the parameter. We present 90\% intervals rather than the standard $95 \%$

668 intervals, as 95\% intervals calculated from simulation draws are less computationally

669 stable [67]. In all cases but the virus phylogenetic effect, the posterior estimates for

670 the proportion of variance explained by each effect differed strongly from their

671 induced priors (Supp Fig 2).

672

673 Summary of Model Results

674 We find evidence that which viral species infects a host, the specific interaction

675 between individual hosts and individual viruses and related hosts having similar

676 prevalences with the same sets of viruses all explain variation in infection prevalence.

677

678 Total Evolutionarily-associated Variation

679 In the models containing the virus phylogeny, approximately a quarter $(25.9 \%$ [11.6-

$68040.4])$ of the total variation in prevalence was explained by terms accounting for the

681 evolutionary histories of hosts and viruses (host phylogenetic effect, virus 
682 phylogenetic effect, host evolutionary interaction effect, virus evolutionary interaction

683 effect and coevolutionary interaction).

684

\section{Host and Virus Level Effects}

686 The host and virus phylogenetic effects measure the extent to which related hosts

687 have similar average prevalences of virus infection and related viruses have similar

688 average prevalences across hosts. The host and virus non-phylogenetic terms measure

689 the extent to which hosts and viruses differ in their average infection levels in manner

690 not consistent with evolution by Brownian motion along a phylogeny. The host

691 species and phylogenetic effects explained a small proportion of the total variance in

692 infection probability (species: $1.9 \%,[0.0-4.6]$; phylogenetic: $2.9 \%,[0.0-6.5])$. The

693 shape of the posterior distributions for the two parameters visualised in Fig 4, makes

694 it clear that the most credible values for both of these parameters are 0 . While it is

695 unlikely that there is no variation in average prevalence between host species, it is

696 clear that the amount of prevalence explained by hosts differing in their infection

697 levels averaged across viruses is small relative to the other effects.

698

699 The virus species and phylogenetic effects explained a larger proportion of the total

700 variance in infection probability, with non-phylogenetic variation dominating, but

701 were imprecisely estimated (species: $13.8 \%$, [3.8-23.7]; phylogenetic: $8.5 \%$, [0.0-

702 17.8]). The posterior density for the phylogenetic effect is concentrated at 0 . So, while

703 it is clear there is a virus species effect, the data does not appear informative for the

704 presence or absence of a viral phylogenetic effect in this system. The posterior draws

705 for the viral species and phylogenetic effect were negatively correlated within

706 iterations, indicating that the model had difficulty partitioning the two. This partial 
non-identifiablility explains the broad posteriors on both.

708

\section{Interaction Effects}

710 A host evolutionary interaction effect measures the extent to which more closely

711 related hosts have more similar prevalences with the same sets of viruses, and a virus

712 evolutionary interaction effect measures the extent to which more closely related

713 viruses infect the same sets of hosts to more similar degrees. A coevolutionary

714 interaction term measures the extent to which more related hosts are infected to

715 similar degrees with viruses that are themselves related. The non-phylogenetic

716 interaction term measures the extent to which there is variance in the mean prevalence

717 of specific host-virus pairings, that are is not consistent with the other interaction 718 effects (see Box 1).

720 There was little evidence for a virus evolutionary interaction effect or coevolutionary

721 interaction having a large effect on the observed prevalences (virus: $2.9 \%$ [0.0-6.1];

722 coevolutionary: $2.0 \%[0.0-4.6])$. In both cases, the marginal posterior distributions 723 peaked at 0 .

725 There was evidence for a host evolutionary interaction explaining some of the total

726 variance in prevalence $(9.5 \%[0.0-18.3])$. This is the only parameter in the model for

727 which the estimated size of the effect depended strongly on the specific treatment of

728 the virus phylogeny (see Fig 4). Whether the lower $90 \%$ bound of the credible interval

729 for the variation explained rounded to 0.0 or 0.1 depended on the phylogenetic matrix

730 (or set of phylogenetic matrices) inputted. The marginal posterior distribution of the

731 parameter was concentrated at lower values when the estimated phylogeny including 
732 the negative-sense RNA viruses was used, and at higher values in the other two cases.

733 However, irrespective of the choice of virus phylogeny, the mode of the distribution

734 and majority of the density was distant from zero, implying that the effect is likely to

735 be biologically relevant. As the virus phylogenies themselves are not actually directly

736 involved in this term, this must be due to the partitioning of variance across other

737 terms being cryptically different depending on the assumptions about the virus

738 phylogeny.

740 There was also a clear non-phylogenetic interaction (15.8\% [5.8-25.4]), implying that

741 much of the variation in prevalence is due to the idiosyncrasies of individual host-

742 virus combinations.

744 As with the virus species effect and the virus phylogenetic effect, the MCMC draws

745 for the proportion of variance explained by the host evolutionary interaction and non-

746 phylogenetic interaction were negatively correlated within an iteration, implying that

747 separating these parameters was proving difficult. While this led to a diffuse posterior

748 with wide credible intervals for both, they remain individually interpretable, and both

749 effects appear present simultaneously. 


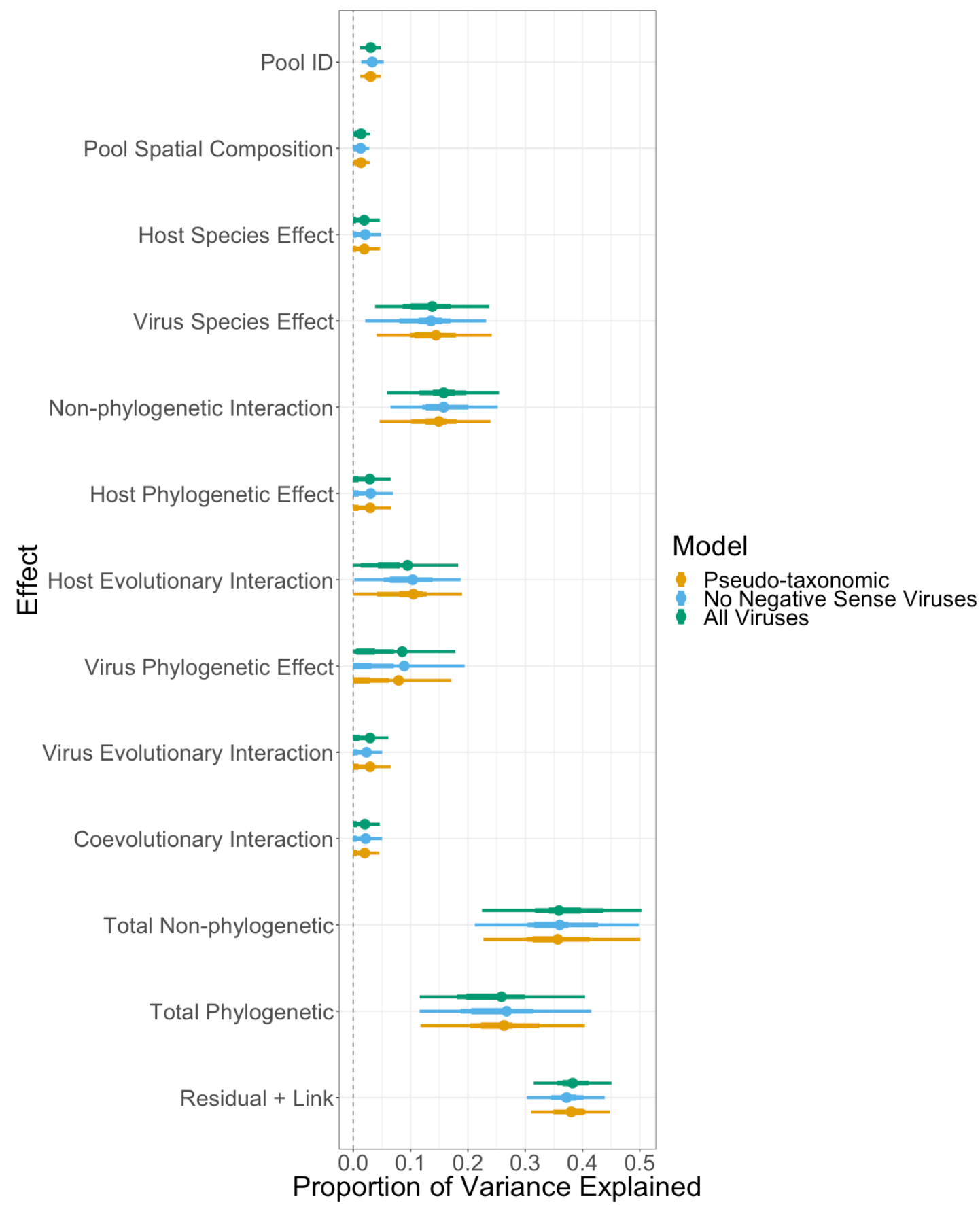

751 Fig 4 Comparison of estimated proportion of variance in prevalence explained by different parameters

752 between models. As each factor explains a proportion of the attributed variance in the model, total

753 variance over all factors must sum to 1. For each parameter, the circle represents the modal estimate,

754 the thick bars represent the $50 \%$ shortest posterior density interval and the thin bars represent the $90 \%$

755 shortest posterior density interval. "Pool ID" is the proportion of the total variation in prevalence

756 explained by pools within species differing in the degree to which they were infected by viruses.

757 "Spatial Composition" is the proportion of the total variation in prevalence explained by the

758 combination of locations from which the bees in the pool originate. "Host Species Effect" is the 
759 proportion of variation in prevalence explained by hosts having different average viral prevalences.

760 "Virus Species Effect" is the proportion of variation in prevalence explained by viruses differing in

761 their average prevalences. "Non-phylogenetic Interaction" is the proportion of variation in prevalence

762 explained by host-virus combinations differing in the their average prevalences beyond that which

763 would be expected by their host and virus species effects alone. "Host Phylogenetic Effect" is the

764 proportion of variation in prevalence explained by hosts having average viral prevalences correlated

765 across the host phylogeny. "Virus Phylogenetic Effect" is the proportion of variation in prevalence

766 explained by viruses having average prevalences correlated across the viral phylogeny. "Host

767 Evolutionary Interaction" is the proportion of variation in prevalence explained by related hosts having

768 correlated viral assemblages. "Virus Evolutionary Interaction" is the proportion of the variation

769 explained by related viruses having correlated host assemblages. "Coevolutionary Interaction" is the

770 proportion of the variation explained by related hosts having similar prevalences of related viruses.

771 "Total Non-phylogenetic" is the proportion of the variation that can be explained by terms not

772 involving the host and virus phylogeny and excluding the residual ("Host Species Effect", "Virus

773 Species Effect”, "Pool ID”, "Spatial Composition Effect”, "Non-phylogenetic Interaction”). "Total

774 phylogenetic" is the proportion of the variation that can be explained by terms involving a host or virus

775 phylogeny ("Host Phylogenetic Effect", "Virus Phylogenetic Effect”, "Host Evolutionary Interaction”,

776 "Virus Evolutionary Interaction", "Coevolutionary Interaction"). "Residual + Link" is the proportion of

777 the total variance that is explained by the residual variance and variance of the logistic distribution

$778\left(\pi^{2} / 3\right)$. 
781 Table 3 Mean estimates for the intra-class correlations of each variance component. The point estimate

782 is the posterior mean, the numbers in brackets represent the $90 \%$ shortest posterior density interval.

\begin{tabular}{c|ccc} 
& All Viruses & No Negative Sense Viruses & Pseudo-taxonomic \\
\hline Virus Species Effect & $13.8(3.8,23.7)$ & $13.6(2.1,23.2)$ & $14.4(4.1,24.2)$ \\
Virus Phylogenetic Effect & $8.5(0,17.8)$ & $8.9(0,19.4)$ & $7.9(0,17.1)$ \\
Host Species Effect & $1.9(0,4.6)$ & $2.1(0,4.8)$ & $1.9(0,4.6)$ \\
Host Phylogenetic Effect & $2.9(0,6.5)$ & $3.0(0,6.9)$ & $2.9(0,6.6)$ \\
Virus Evolutionary Interaction & $2.9(0,6.1)$ & $2.3(0,5.1)$ & $2.9(0,6.6)$ \\
Host Evolutionary Interaction & $9.5(0,18.3)$ & $10.4(0.2,18.8)$ & $10.5(0,19.0)$ \\
Non-phylogenetic Interaction & $15.8(5.8,25.4)$ & $15.8(6.5,25.2)$ & $14.9(4.6,24.0)$ \\
Coevolutionary Interaction & $2.0(0,4.6)$ & $2.2(0,5.0)$ & $2.0(0,4.5)$ \\
Pool ID & $3.0(1.1,4.8)$ & $3.3(1.4,5.3)$ & $3.0(1.2,4.8)$ \\
Pool Spatial Composition & $1.4(0,2.9)$ & $1.3(0,2.8)$ & $1.3(0,2.9)$ \\
Residual + Link & $38.3(31.5,45.1)$ & $37.2(30.3,43.9)$ & $38(31,44.7)$ \\
Total Non-phylogenetic & $35.9(22.4,50.3)$ & $36(21.2,49.8)$ & $35.7(22.7,50.1)$ \\
Total Phylogenetic & $25.9(11.6,40.4)$ & $26.8(11.6,41.5)$ & $26.3(11.7,40.4)$
\end{tabular}

\section{Tongue Length-Viral Community Correlation}

786 Given that the co-phylogenetic model found that related hosts share viral

communities, one potential mechanism for this is phylogenetically-biased exposure,

788 driven by phylogenetically correlated floral preferences. If bumblebee species with

789 similar flower preferences had similar viral communities, it would be expected that

790 there would be a positive correlation between tongue length similarity (as this is an

791 important factor in floral preference) and viral community similarity between pairs of

792 species. The point estimate of the correlation between the two distances was small

793 and negative (-0.06), but the 95\% confidence intervals for that point estimate

794 overlapped zero $(-0.13,0.00)$, so given the uncertainty in the data, a correlation of

795 zero cannot be rejected. Nonetheless, given this data, a strong positive relationship

796 between tongue length and viral community similarity seems unlikely, a result 
inconsistent with phylogenetically-biased exposure driven by tongue length-mediated floral choice.

\section{Discussion}

801 Using wild bumblebee species that share transmission opportunities, we have shown that variation in the prevalence of infection in the wild is explained by related hosts being infected with the same viruses to similar degrees, viruses differing in their average prevalence and individual virus-host pairings having greater or lesser prevalence than would be expected by the host and virus species effects alone.

\section{Virus Discovery}

808 There is now an extensive diversity of viruses known in bees, with most new studies

809 finding novel viruses [30,71,73,74,85-87]. We have found 37 novel putative viral

810 contigs in the transcriptomes of wild-caught bumblebees from across Scotland,

811 suggesting that virus discovery in this taxonomic group is far from saturation. As with

812 any metagenomic study, it is hard to be confident that the virus-like contigs represent 813 real infections of the sampled host, rather than surface or gut contaminants. However, 814 the presence of 22nt virus siRNAs, generated from double-stranded viruses by Dicer 815 as part of an antiviral response in the host, provides compelling evidence that at least 81610 of these contigs (Densovirus 2 and 3, Elf Loch virus, Allermuir Hill virus 1, 2 and 817 3, Mill Lade virus, Mayfield virus 1 and 2, and Castleton Burn virus) represent active 818 viral infections in bumblebees.

820 Mites and nematodes both parasitise bumblebees and therefore could potentially be an 821 alternative source of the small RNAs. Mite viRNAs are reported to be centered at 
822

823

824

826

24nt [73], and could therefore not produce the small RNA patterns observed.

Nematode viRNAs are centered at 22nt, like bumblebee viRNAs, [88] and thus could potentially produce this pattern. While, outside of queens infected with Sphaerularia bombi, nematode infection of wild bumblebees appears to be very rare [89], nematodes cannot be categorically ruled out as a source of the observed small RNAs. One contig's (MH614312) mapped small RNAs were centered at 21nt, and the closest known virus was a nepovirus of plants. As DCL4, a major plant Dicer, produces viRNAs of this size [90], this is consistent with that particular virus being a plant virus, which was transferred in collected nectar or pollen.

\section{Phylogenetic Effects}

We found no evidence for a large host phylogenetic effect, where related hosts have correlated average viral prevalences. To the best of our knowledge no studies have previously applied these methods to viruses sampled from wild animals. However, other traits relating to viral disease in a series of studies in Drosophila species under experimental conditions have consistently detected host phylogenetic effects in factors that would be expected to be correlated with prevalence in the wild, such as infection probability [24], virulence and viral load [91] and viral load alone [92]. However, two of these studies focused on a single isolate of Drosophila C virus. Therefore, the variation that they attribute to a phylogenetic effect may be partitioned into the host evolutionary interaction in our study, as a host evolutionary interaction is equivalent to separate inconsistent host phylogenetic effects for each virus. Our data were not particularly informative for the presence or absence of a virus phylogenetic effect, with the posterior being very diffuse with a majority of the density near zero. This appears partly due to difficulties partitioning the variation between the virus 
species effect and virus phylogenetic effect. Irrespective of the cause, we can make no hosts from this dataset.

\section{Host Species Effect}

852 There was little evidence for an important effect of host species independent of the 853 phylogeny, implying that hosts do not strongly vary in the average degree to which

854 they are infected with viruses. The previous studies using these methods have

855 universally found host species effects $[25,93]$. However, as both of these studies have

856 used mammal-eukaryotic parasite datasets, the degree of relevance for them as a

857 comparison in unclear. Experimental evidence from virus studies across drosophilid

858 flies have found weak to zero host species effects on the titre of sigma viruses [24]

859 and Drosophila C virus virulence [91] but considerably larger host species effects on

860 Drosophila C virus load [91]. This between-study variation potentially indicates a

861 reason we did not detect a host species effect. With a single pathogen, the average and

862 particular degrees of variation in infection between hosts are identical. As soon as

863 multiple pathogens are involved, they diverge, such that it is possible for there to be

864 no variation in the average prevalence between hosts, but still considerable variation

865 in the prevalence of particular viruses between hosts, which is consistent with the

866 presence of a host species effect in correlates of prevalence in some viruses but not

867 others, as noted above.

\section{$869 \quad$ Virus Species Effect}

870 A clear effect of virus species independent of the virus phylogeny was detected in the

871 dataset, despite the uncertainty added by the difficulty partitioning the virus species 
872 and phylogenetic effects. Therefore, viruses differed in their prevalences averaged

873 across hosts. This is an intuitive result as viruses differ in host range [94], virulence

$874[95,96]$ and infectious period [96] at both the species and the strain level. Variation in

875 host range changes the size of the host pool available for infection, and variation in

876 virulence and infectious period both change the length of time any infected host is

877 available for sampling. All these factors would be expected to drive consistent

878 differences in long-run prevalences between viruses. Additionally, as our sites were

879 only sampled once, short-term effects will also drive between virus variation. Any

880 virus that was experiencing an epizootic at the time of sampling will be

881 overrepresented relative to its long-run prevalence, further increasing the between

882 virus variation.

883

\section{Host Evolutionary Interaction Effect}

885 A host assemblage effect was found, where phylogenetically related hosts share viral

886 assemblages, showing that more closely related hosts are more similar in virus

887 prevalence for groups of viruses. The statistical machinery required for estimating this

888 effect is quite new and, in the disease ecology field, has predominantly been applied

889 to mammal-parasite and plant-parasite systems where there are good datasets already.

890 Nonetheless, host evolutionary interactions have always been found when searched

891 for using these methods [25,93] and analogous effects are commonly found using

892 different methods [97-99]. In a system where these viruses were host limited, this

893 pattern could be explained by preferential host shifting, where parasites more

894 frequently gain the ability to infect hosts closely related to ones that they are already

895 capable of infecting. Preferential host shifting is known to be a general phenomenon,

896 and has been observed in macroparasites, viruses and protozoans (see Longdon et al 
(2014) [27] and the references within).

898

899 While some of the viruses in this study were not detected in a subset of host species,

900 most of the viruses found here appear to genuinely be multihost viruses, with the

901 majority being detected in over half the sampled species. Given this, a combination of

902 biased cross-species transmission and preferential host shifting appears a better

903 explanation in this system. Biased cross-species transmission occurs when

904 transmission occurs more frequently between some species that a pathogen is already

905 capable of infecting than others. This biased cross-species transmission could be

906 driven by two non-exclusive mechanisms: phylogenetically-biased transmission

907 probabilities and phylogenetically-biased exposure.

908

909 Phylogenetically-biased transmission probabilities occur when cross-species

910 transmission is more frequent among close relatives, due to the probability of

911 infection after contact with the virus being similar between related species. Related

912 hosts present correlated environments from the perspective of the virus at the

913 molecular and anatomical level, therefore adaptation to one should provide

914 corresponding fitness increases on the other. Experimental results have shown that

915 correlated mutations occur on viral entry to related hosts [100], implying that this

916 cross-adaptation does occur. However, this is probabilistic, and the mutations fixed on

917 entry can differ between replicate entries [100,101]. Therefore, if there is antagonistic

918 pleiotropy between mutations that are adaptive in two different groups of hosts and

919 cross-adaptation predicts the probability of successful infection on contact, then a

920 phylogenetically-biased transmission network will result. 
922 Phylogenetically-biased exposure represents an evolutionarily-driven ecological

923 phenomenon that biases cross-species transmission rates, mediated by niche overlap.

924 Contaminated flowers are likely to be an important source of intra- and inter- specific

925 pathogen transmission in bumblebees and pollinators more generally [102-104]. The

926 flower visitation network has been shown to be associated with the partitioning of

927 genetic diversity of Crithidia bombi between bumblebee hosts [9], and the network

928 itself is highly structured, though temporally variable [105]. Different bumblebee

929 species show tongue length differences, which are phylogenetically associated [15],

930 and the differences in tongue length correlate with differential flower usage between

931 bumblebee species $[12,106]$. If infection occurs at contaminated flowers, the

932 structuring of the flower usage network could cause different flowers to build up

933 different surface viral communities. This could drive consistent phylogenetically-

934 correlated differences in viral infection rates through differential exposure.

936 Post-hoc testing did not find a positive relationship between tongue length

937 dissimilarity (a rough proxy for species-level flower choice dissimilarity) and viral

938 community composition dissimilarity, which provides some evidence against

939 phylogenetically-biased exposure as the causative mechanism. However, the study

940 design in this case is not optimal for disentangling biased transmission probabilities

941 and biased exposure, as species were sampled from different locations at a single

942 timepoint and prevalence of the viruses varied spatially. Given this, drawing strong

943 conclusions as to the relative impact of the two mechanisms outlined above based on

944 this data would be premature. Similarly, the subgenus Psithyrus contains socially

945 parasitic species that are coevolved to parasitise particular social bumblebee species,

946 which could also lead to phylogenetically-correlated differences in viral infection 
rates. We were unable to test whether socially parasitic cuckoo bumblebee species

948 have similar viral communities to their hosts, as our study included only a single

949 parasitic bumblebee species, B. bohemicus, but the possibility of brood parasitism

950 being an important driver of between-colony disease transmission is worth further

951 study.

952

\section{Virus Evolutionary Interaction Effect and Coevolutionary Interaction}

954 We found no evidence of a large virus evolutionary interaction or coevolutionary

955 interaction. This is largely unsurprising as it would appear implausible that the host

956 assemblages have been conserved over evolutionary time, as the deep splits in the

957 viral families predate the most recent common ancestor of bumblebees by many

958 millions of years [107].

959

\section{Non-phylogenetic Interaction}

961 A non-phylogenetic interaction was detected. This interaction represents variation in

962 prevalence caused by specific host-virus pairings having prevalences beyond that

963 which would be expected by the simple addition of the individual host and virus

964 means. A non-phylogenetic interaction could be caused by a large range of factors,

965 some biological and some due to the specifics of the model, many of which would be

966 likely to be acting simultaneously to generate this signal. One possibility is

967 coevolution between the host and virus that occurred after both diverged from their

968 common ancestor with the closest related species in the study. Another is the

969 complete absence of coevolution, where spillover from a primary or group of primary

970 hosts causes either a constant very low prevalence of dead-end infections, which are

971 none-the-less detectable by PCR. Related to this is a statistical issue involving cases 
972 where not every species in the study is within a virus' host range, and the species that

973 are within the host range are not closely related. In this case, the variation is not

974 absorbed by the host evolutionary interaction and almost no host has a prevalence

975 close to the mean across hosts, as in many species the prevalence is zero, which

976 causes the mean to be considerably lower than the average prevalence in the species

977 the virus does infect. This effect would be magnified if the sampling occurs during an

978 epizootic. More broadly, anything that changes the epidemiological parameters of a

979 virus in a specific host will lead to a non-phylogenetic interaction. Considering the

980 variation in the natural history of viruses and the lesser, but still significant, variation

981 in the natural history of bumblebees, a large non-phylogenetic interaction is to be

982 expected.

983

984 Conclusion

985 While it is clear that viruses are abundant in pollinators, the factors that determine the

986 distribution of pollinator viruses have remained uncertain, outside of a few well-

987 studied cases $[108,109]$. With the novel viruses discovered in this study, we have

988 investigated predictors of these virus/host associations and found that both the host

989 evolutionary history and the identity of the virus contributes to this distribution. This

990 supports both theory and prior empirical evidence that related species are more at risk

991 of infection from each other's diseases than the diseases of distantly related species.

992 However, the importance of the viral identity and unique interactions between host-

993 virus pairs suggests that the introduction of a novel virus into a community is likely to

994 have unpredictable effects even when no close relatives of currently known hosts are

995 present. This highlights the risk posed by disease spillover for the conservation not 
only of wild pollinator communities, but also to communities consisting of related

997 animal or plant species in general.

998

\section{Data Availability}

1000 The data and code for running the analyses is available on github under a GPLv3

1001 licence, as code uses code taken from other GPLv3 licenced works

1002 (https://github.com/dpascall/bumblebee-virus-cophylo).

1003

1004 Acknowledgements

1005 We thank Jarrod Hadfield for extensive statistical advice, modifications to

1006 MCMCglmm and for helpful comments on this manuscript, Dave Goulson for

1007 assistance identifying some specimens and to Ben Longdon and Bethany Clark for

1008 further comments. Rowan Doff assisted in the lab with RFLP analyses and Claire

1009 Webster with DNAse treatments. Claire Webster, Jarrod Hadfield and Florian Bayer

1010 helped with fieldwork.

1011

$1012 \quad$ Funding

1013 This work was funded by a BBSRC SWBIO DTP PhD stipend to DP, a Royal Society

1014 Dorothy Hodgkin Fellowship to LW, Wellcome Trust Research Career Development

1015 Fellowship WT085064 to DJO.

1016

1017 References

1018 1. Woolhouse ME, Gowtage-Sequeria S. Host range and emerging and

1019 reemerging pathogens. Emerg Infect Dis. 2005;11(12): 1842-7.

1020 2. Suttle CA. Marine viruses--major players in the global ecosystem. Nat Rev 
$1021 \quad$ Microbiol. 2007;5(10): 801-12.

1022 3. Williams PH, Osborne JL. Bumblebee vulnerability and conservation world-

$1023 \quad$ wide. Apidologie. 2009;40(3): 367-87

1024 4. Garibaldi LA, Steffan-Dewenter I, Winfree R, Aizen MA, Bommarco R,

1025 Cunningham SA, et al. Wild pollinators enhance fruit set of crops regardless of 1026 honey bee abundance. Science. 2013;340(6127): 1608-11.

1027 5. Vanbergen AJ, the Insect Pollinators Initiative. Threats to an ecosystem

1028 service: Pressures on pollinators, Front Ecol Environ. 2013;11(5): 251-259.

1029 6. Manley R, Boots M, Wilfert L. Emerging viral disease risk to pollinating

1030 insects: Ecological, evolutionary and anthropogenic factors. J Appl Ecol.

$1031 \quad 2015 ; 52(2): 331-40$.

1032 7. Fenton A, Streicker DG, Petchey OL, Pedersen AB. Are all hosts created

1033 equal? Partitioning host species contributions to parasite persistence in

1034 multihost communities. Am Nat. 2015;186(5): 610-22.

1035 8. Heesterbeek JA. A brief history of R0 and a recipe for its calculation. Acta $1036 \quad$ Biotheor. 2002;50: 189-204.

1037 9. Salathé RM, Schmid-Hempel P. The genotypic structure of a multi-host 1038 bumblebee parasite suggests a role for ecological niche overlap. PLoS One. $1039 \quad 2011 ; 6(8):$ e22054.

1040 10. Arbulo, N, Santos, E, Salvarrey, S, \& Invernizzi C. Proboscis length and 1041 resource utilization in two ruguayan bumblebees: Bombus atratus Franklin and 1042 Bombus bellicosus Smith (Hymenoptera: Apidae). Neotrop Entomol. $1043 \quad 2011 ; 40(4): 483-8$.

1044 11. Goulson D, Darvill B. Niche overlap and diet breadth in bumblebees; are rare 1045 species more specialized in their choice of flowers? Apidologie. 2004;35(1): 
$55-63$.

1047 12. Goulson D, Lye GC, Darvill B. Diet breadth, coexistence and rarity in

1048 bumblebees. Biodivers Conserv. 2008;17(13): 3269-88

1049 13. Harder LD. Morphology as a predictor of flower choice by bumble bees.

$1050 \quad$ Ecology. 1985;66(1): 198-210.

1051 14. Lye GC, Kaden JC, Park KJ, Goulson D. Forage use and niche partitioning by 1052 non-native bumblebees in New Zealand: Implications for the conservation of 1053 their populations of origin. J Insect Conserv. 2010;14(6): 607-15.

1054 15. Harmon-Threatt AN, Ackerly DD. Filtering across spatial scales: Phylogeny, 1055 biogeography and community structure in bumble bees. PLoS One. 2013;8(3): $1056 \quad$ e60446.

1057 16. Goodwin SG. Seasonal phenology and abundance of early-, mid- and long1058 season bumble bees in southern England. J Apic Res. 1995;34(2): 79-87.

1059 17. van Riper III C, van Riper SG, Goff ML, Laird M. The epizootiology and 1060 ecological significance of malaria in Hawaiian land birds. Ecol Monogr. $1061 \quad$ 1986;56(4): 327-44.

1062 18. Hueffer K, Parker JS, Weichert WS, Geisel RE, Sgro JY, Parrish CR. The natural host range shift and subsequent evolution of canine parvovirus resulted 1064 from virus-specific binding to the canine transferrin receptor. J Virol. $1065 \quad 2003 ; 77(3): 1718-26$.

1066 19. Johnson MB, Lafferty KD, van Oosterhout C, Cable J. Parasite transmission in 1067 social interacting hosts: Monogenean epidemics in guppies. PLoS One. 2011;6(8): e22634.

1069 20. Arneberg P, Skorping A, Grenfell B, Read AF. Host densities as determinants 1070 of abundance in parasite communities. Proc R Soc B Biol Sci. 1998;265(1403): 
$1283-9$.

1072 21. Civitello DJ, Cohen J, Fatima H, Halstead NT, Liriano J, Mcmahon TA, et al. Acad Sci U S A. 2015;112(28): 8667-71.

22. Curtis VA. Infection-avoidance behaviour in humans and other animals. Trends Immunol. 2014;35(10): 457-64.

23. Chandra RK. Nutrition, immunity, and infection: present knowledge and future directions. Lancet. 1983;321(8326): 688-91.

24. Longdon B, Hadfield JD, Webster CL, Obbard DJ, Jiggins FM. Host phylogeny determines viral persistence and replication in novel hosts. PLoS Pathog. 2011;7(9): e1002260.

25. Hadfield JD, Krasnov BR, Poulin R, Nakagawa S. A tale of two phylogenies:

26. Rafferty NE, Ives AR. Phylogenetic trait-based analyses of ecological networks. Ecology. 2013;94(10): 2321-33.

27. Longdon B, Brockhurst MA, Russell CA, Welch JJ, Jiggins FM. The evolution and genetics of virus host shifts. PLoS Pathog. 2014;10(11): e1004395.

28. Bailey L, Woods RD. Three previously undescribed viruses from the honey bee. J Gen Virol. 1974;25(2): 175-86.

1091 29. Bailey L, Gibbs AJ, Woods RD. Two viruses from adult honey bees (Apis mellifera Linnaeus). Virology. 1963;21(3): 390-5.

1093 30. Cornman RS, Tarpy DR, Chen Y, Jeffreys L, Lopez D, Pettis JS, et al. 1094 Pathogen webs in collapsing honey bee colonies. PLoS One. 2012;7(8): $1095 \quad$ e43562. 
1096

1097

1098

1099

1100

1101

1102

1103

1104

1105

1106

1107

1108

1109

1110

1113

1114

1115

1116

1117

1118

1120

31. Shi M, Lin XD, Tian JH, Chen LJ, Chen X, Li CX, et al. Redefining the invertebrate RNA virosphere. Nature. 2016;540(7634): 539-543.

32. Murray TE, Fitzpatrick Ú, Brown MJF, Paxton RJ. Cryptic species diversity in a widespread bumble bee complex revealed using mitochondrial DNA RFLPs. Conserv Genet. 2008;9(3): 653-666.

33. Langmead B, Salzberg SL. Fast gapped-read alignment with Bowtie 2. Nat Methods. 2012;9(4): 357-9.

34. Grabherr MG, Haas BJ, Yassour M, Levin JZ, Thompson DA, Amit I, et al. Full-length transcriptome assembly from RNA-Seq data without a reference genome. Nat Biotechnol. 2011;29(7): 644-52.

35. Buchfink B, Xie C, Huson DH. Fast and sensitive protein alignment using DIAMOND. Nat Methods. 2015;12(1): 59-60.

36. Altschul SF, Gish W, Miller W, Myers EW, Lipman DJ. Basic local alignment search tool. J Mol Biol. 1990;215(3): 403-10.

37. Fauquet CM, Stanley J. Revising the way we conceive and name viruses below the species level: A review of geminivirus taxonomy calls for new standardized isolate descriptors. Arch Virol. 2005;150: 2151-2179.

38. Untergasser A, Cutcutache I, Koressaar T, Ye J, Faircloth BC, Remm M, et al. Primer3-new capabilities and interfaces. Nucleic Acids Res. 2012;40(15): e115.

39. Cameron SA, Hines HM, Williams PH. A comprehensive phylogeny of the bumble bees (Bombus). Biol J Linn Soc. 2007;91(1): 161-88.

40. Katoh K, Rozewicki J, Yamada KD. MAFFT online service: multiple sequence alignment, interactive sequence choice and visualization. Brief Bioinform. 2017. doi: 10.1093/bib/bbx108 
1121 41. Katoh K, Kuma K, Toh H, Miyata T. MAFFT version 5: improvement in accuracy of multiple sequence alignment. Nucleic Acids Res. 2005;33(2): 511518.

42. $\quad$ Bouckaert R, Heled J, Kühnert D, Vaughan T, Wu CH, Xie D, et al. BEAST 2: A software platform for Bayesian evolutionary analysis. PLoS Comput Biol. 2014;10(4): e1003537.

43. Bouckaert RR, Drummond AJ. bModelTest: Bayesian phylogenetic site model averaging and model comparison. BMC Evol Biol. 2017;17(1): 42.

44. Heled J, Drummond AJ. Calibrated tree priors for relaxed phylogenetics and divergence time estimation. Syst Biol. 2012;61(1): 138-49. dating with confidence. PLoS Biol. 2006;4(5): 699-710.

46. Hines HM. Historical biogeography, divergence times, and diversification patterns of bumble bees (Hymenoptera: Apidae: Bombus). Syst Biol. 2008;57(1): 58-75. Available from: http://beast.bio.ed.ac.uk/Tracer

48. Kanehisa M, Goto S, Kawashima S, Nakaya A. The KEGG databases at GenomeNet. Nucleic Acids Res. 2002;30(1): 42-46.

1140 49. Finn RD, Bateman A, Clements J, Coggill P, Eberhardt RY, Eddy SR, et al. 1141 Pfam: The protein families database. Nucleic Acids Res. 2014;42(D1): D222D230.

1143 50. Kamer G, Argos P. Primary structural comparison of RNA-dependent 1144 polymerases from plant, animal and bacterial viruses. Nucleic Acids Res. $1145 \quad$ 1984;12(18): 7269-82. 
1146 51. Pei J, Kim BH, Grishin NV. PROMALS3D: A tool for multiple protein

1147 sequence and structure alignments. Nucleic Acids Res. 2008;36(7): 2295-300.

1148 52. Koonin EV, Dolja VV, Krupovic M. Origins and evolution of viruses of 1149 eukaryotes: The ultimate modularity. Virology. 2015;79-80: 2-25.

1150 53. Zanotto PM, Gibbs MJ, Gould EA, Holmes EC. A reevaluation of the higher 1151 taxonomy of viruses based on RNA polymerases. J Virol. 1996;70(9): 60836096.

1153 54. Gorbalenya AE, Pringle FM, Zeddam JL, Luke BT, Cameron CE, Kalmakoff J, 1154 et al. The palm subdomain-based active site is internally permuted in viral RNA-dependent RNA polymerases of an ancient lineage. J Mol Biol. 2002;324(1): 47-62.

1157 55. Henikoff S, Henikoff JG. Amino acid substitution matrices from protein blocks. Proc Natl Acad Sci U S A. 1992;89(22): 10915-10919.

1159 56. Ferreira MAR, Suchard MA. Bayesian analysis of elapsed times in continuous1160 time Markov chains. Can J Stat. 2008;36(3): 355-68.

1161 57. Holmes EC. Molecular clocks and the puzzle of RNA virus origins. J Virol. 2003;77(7): 3893-3897.

1163 58. Katzourakis A, Gifford RJ. Endogenous viral elements in animal genomes.

$1164 \quad$ PLoS Genet. 2010;6(11): e1001191.

1165 59. Chiba S, Kondo H, Tani A, Saisho D, Sakamoto W, Kanematsu S, et al.

1166 Widespread endogenization of genome sequences of non-retroviral RNA $1167 \quad$ viruses into plant genomes. PLoS Pathog. 2011;7(7): e1002146.

1168 60. Drummond AJ, Suchard MA, Xie D, Rambaut A. Bayesian phylogenetics with 1169 BEAUti and the BEAST 1.7. Mol Biol Evol. 2012;29(8): 1969-1973.

1170 61. Webster CL, Waldron FM, Robertson S, Crowson D, Ferrari G, Quintana JF, et 
al. The discovery, distribution, and evolution of viruses associated with

1173 62. Ebert TA, Brlansky R, Rogers M. Reexamining the pooled sampling approach for estimating prevalence of infected insect vectors. Ann Entomol Soc Am. 2010;103(6): 827-37.

63. Gibbs AJ, Gower JC. The use of a multiple-transfer method in plant virus transmission studies - Some statistical points arising in the analysis of results. Ann Appl Biol. 1960;48(1): 75-83.

64. Thompson KH. Estimation of the proportion of vectors in a natural population

1181 65. Carpenter B, Gelman A, Hoffman MD, Lee D, Goodrich B, Betancourt M, et al. Stan: A probabilistic programming language. J Stat Softw. 2017;76(1): 132.

66. Liu Y, Gelman A, Zheng T. Simulation-efficient shortest probability intervals. Stat Comput. 2015;25(4): 809-819.

1186 67. Stan Development Team. Stan Modeling Language: User's Guide and Reference Manual. 2.17.1. 2017. Available from: https://github.com/stan$\mathrm{dev} / \mathrm{stan} /$ releases/download/v2.17.1/stan-reference-2.17.1.pdf.

1189 68. Goulson D, Hanley ME, Darvill B, Ellis JS, Knight ME. Causes of rarity in $1190 \quad$ bumblebees. Biol Conserv. 2005;122(1): 1-8.

1191 69. Bailey L, Ball BV. Honey bee pathology. 2nd ed. London: Academic Press $1192 \quad$ Inc.; 1991.

1193 70. de Miranda JR, Cornman RS, Evans JD, Semberg E, Haddad N, Neumann P, et 1194 al. Genome characterization, prevalence and distribution of a macula-like virus 1195 from Apis mellifera and Varroa destructor. Viruses. 2015;7(7): 3586-602. 
1196 71. Schoonvaere K, Smet LD, Smagghe G, Vierstraete A, Braeckman BP, de Graaf DC, et al. Unbiased RNA shotgun metagenomics in social and solitary wild bees detects associations with eukaryote parasites and new viruses. PLoS One. 2016;11(12): e0168456.

1200 72. Bailey L, Woods RD. Two more small RNA viruses from honey bees and further observations on sacbrood and acute bee-paralysis viruses. J Gen Virol. 1977;37(1): 175-82.

73. Remnant EJ, Shi M, Buchmann G, Blacquière T, Holmes EC, Beekman M, et al. A diverse range of novel RNA viruses in geographically distinct honey bee populations. J Virol. 2017; 91(16): e00158-17. seasonal prevalence of known viruses, Nosema, and Crithidia. PLoS One. 2011;6(6): e20656.

75. Boccardo G, Milne RG, Luisoni E, Lisa V, Accotto GP. Three seedborne cryptic viruses containing double-stranded RNA isolated from white clover. Virology. 1985;147(1): 29-40.

1213 76. Smith KM, Markham R. Two new viruses affecting tobacco and other plants. 1214 Phytopathology. 1944;34: 324-329.

1215 77. Koloniuk I, Přibylová J, Fránová J. Molecular characterization and complete 1216 genome of a novel nepovirus from red clover. Arch Virol. 2018;163(5): 13879.

1218 78. Bronkhorst AW, van Cleef KW, Vodovar N, İnce İA, Blanc H, Vlak JM, et al. The DNA virus Invertebrate iridescent virus 6 is a target of the Drosophila RNAi machinery. Proc Natl Acad Sci U S A. 2012;109(51): E3604-13. 
1221 79. Brennecke J, Aravin AA, Stark A, Dus M, Kellis M, Sachidanandam R, et al. Discrete small RNA-generating loci as master regulators of transposon activity in Drosophila. Cell. 2007;128(6): 1089-103.

1224 80. Suzuki Y, Frangeul L, Dickson LB, Blanc H, Verdier Y, Vinh J, et al. Uncovering the repertoire of endogenous flaviviral elements in Aedes. J Virol. 2017;91(15): e00571-17.

81. Lewis SH, Quarles KA, Yang Y, Tanguy M, Frézal L, Smith SA, et al. Panarthropod analysis reveals somatic piRNAs as an ancestral defence against transposable elements. Nat Ecol Evol. 2018;2(1): 174-81.

1230 82. van Rij RP, Saleh MC, Berry B, Foo C, Houk A, Antoniewski C, et al. The RNA silencing endonuclease Argonaute 2 mediates specific antiviral immunity in Drosophila melanogaster. Genes Dev. 2006;20(21): 2985-95.

83. van Mierlo JT, Bronkhorst AW, Overheul GJ, Sadanandan SA, Ekström JO, Heestermans M, et al. Convergent evolution of Argonaute-2 Slicer antagonism

1236 84. Deleris A, Gallago-Bartolome J, Bao J, Kasschau KD, Carrington JC, Voinnet O. Hierarchical action and inhibition of plant dicer-like proteins in antiviral defense. Science. 2006;313(5783): 68-71.

85. Mordecai GJ, Wilfert L, Martin SJ, Jones IM, Schroeder DC. Diversity in a honey bee pathogen: first report of a third master variant of the Deformed

1242 86. Schoonvaere K, Smagghe G, Francis F, de Graaf DC. Study of the metatranscriptome of eight social and solitary wild bee species reveals novel viruses and bee parasites. Front Microbiol. 2018. doi: 
1246 87. Roberts JMK, Anderson DL, Durr PA. Metagenomic analysis of Varroa-free Australian honey bees (Apis mellifera) shows a diverse Picornavirales virome. J Gen Virol. 2018;99: 818-26.

88. Félix MA, Ashe A, Piffaretti J, Wu G, Nuez I, Bélicard T, et al. Natural and experimental infection of Caenorhabditis nematodes by novel viruses related to nodaviruses. PLoS Biol. 2011;9(1): e1000586.

89. Rao S, Poinar G, Henley D. A scientific note on rare parasitism of the bumble bee pollinator, Bombus impatiens, by a mermithid nematode, Pheromermis $\mathrm{sp}$. (Nematoda: Mermithidae). Apidologie. 2017;48(1): 75-7.

1255 90. Wang XB, Jovel J, Udomporn P, Wang Y, Wu Q, Li WX, et al. The 21nucleotide, but not 22-nucleotide, viral secondary small interfering RNAs direct potent antiviral defense by two cooperative Argonautes in Arabidopsis thaliana. Plant Cell. 2011;23(4): 1625-38.

91. Longdon B, Hadfield JD, Day JP, Smith SC, McGonigle JE, Cogni R, et al. The causes and consequences of changes in virulence following pathogen host shifts. PLoS Pathog. 2015;11(3): e1004728.

1262 92. Roberts K, Hadfield JD, Sharma MD, Longdon B. Changes in temperature alter susceptibility to a virus following a host shift. PLoS Patho. 2018;14(10): e1007185

1265 93. Waxman D, Weinert LA, Welch JJ. Inferring host range dynamics from comparative data: The protozoan parasites of New World monkeys. Am Nat. 2014;184(1): 65-74.

1268 94. Bandín I, Dopazo CP. Host range, host specificity and hypothesized host shift events among viruses of lower vertebrates. Vet Res. 2011;42(67).

1270 95. Langsjoen RM, Haller SL, Roy CJ, Vinet-Oliphant H, Bergren NA, Erasmus 
$\mathrm{JH}$, et al. Chikungunya virus strains show lineage-specific variations in virulence and cross-protective ability in murine and nonhuman primate models. MBio. 2018;9(2): e02449-17.

1274 96. Baker C, Antonovics J. Evolutionary determinants of genetic variation in susceptibility to infectious diseases in humans. PLoS One. 2012; 7(1): e29089.

1276 97. Davies TJ, Pedersen AB. Phylogeny and geography predict pathogen community similarity in wild primates and humans. Proc R Soc B Biol Sci. 2008;275(1643): 1695-701.

98. Huang S, Bininda-Emonds OR, Stephens PR, Gittleman JL, Altizer S. Phylogenetically related and ecologically similar carnivores harbour similar parasite assemblages. J Anim Ecol. 2014;83(3): 671-80.

99. Cooper N, Griffin R, Franz M, Omotayo M, Nunn CL, Fryxell J. Phylogenetic host specificity and understanding parasite sharing in primates. Ecol Lett. 2012;15(12): 1370-7.

100. Longdon B, Day JP, Alves JM, Smith SCL, Houslay TM, McGonigle JE, et al. Host shifts result in parallel genetic changes when viruses evolve in closely related species. PLoS Pathog. 2018;14(4): e1006951.

101. Streicker DG, Altizer SM, Velasco-Villa A, Rupprecht CE. Variable evolutionary routes to host establishment across repeated rabies virus host shifts among bats. Proc Natl Acad Sci U S A. 2012;109(48): 19715-19720.

1291 102. Durrer S, Schmid-Hempel P. Shared use of flowers leads to horizontal pathogen transmission. Proc R Soc B Biol Sci. 1994;258(1353): 299-302.

1293 103. Graystock P, Goulson D, Hughes WO. Parasites in bloom: flowers aid dispersal and transmission of pollinator parasites within and between bee species. Proc R Soc B Biol Sci. 2015;282(1813): 20151371. 
104. McArt SH, Koch H, Irwin RE, Adler LS. Arranging the bouquet of disease: floral traits and the transmission of plant and animal pathogens. Ecology Letters. 2014;17(5): 624-36.

105. Ruiz-González MX, Bryden J, Moret Y, Reber-Funk C, Schmid-Hempel P, Brown MJ. Dynamic transmission, host quality, and population structure in a multihost parasite of bumblebees. Evolution. 2012;66(10): 3053-66.

106. Inouye DW. Resource partitioning in bumblebees: Experimental studies of foraging behavior. Ecology. 1978;59(4): 672-8.

1304 107. Koonin EV, Wolf YI, Nagasaki K, Dolja VV. The Big Bang of picorna-like 1305 virus evolution antedates the radiation of eukaryotic supergroups. Nat Rev Microbiol. 2008;6(12): 925-39.

1307 108. Fürst MA, McMahon DP, Osborne JL, Paxton RJ, Brown MJ. Disease associations between honeybees and bumblebees as a threat to wild pollinators. Nature. 2014;506(7488): 364-366.

1310 109. McMahon DP, Fürst MA, Caspar J, Theodorou P, Brown MJF, Paxton RJ. A sting in the spit: Widespread cross-infection of multiple RNA viruses across wild and managed bees. J Anim Ecol. 2015;84(3): 615-24. 